\title{
Mineralogy and Magmatic Processes of Cenozoic Intraplate Alkaline Volcanic Rocks of Bafang and Its Environs (Cameroon Volcanic Line, West Africa)
}

\author{
Nicaise Blaise Tchuimegnie Ngongang1*, Merlin Patrick Njombie Wagsong1*, \\ François Mvondo Owono', Innocent Badriyo ${ }^{2,3}$, Philippe Essomba ${ }^{3}$, \\ Natalie Love Ngongang Tchikankou ${ }^{4}$, Dieudonné Youmen ${ }^{1}$, Pierre Kamgang ${ }^{3}$, Gilles Chazot $^{5}$ \\ ${ }^{1}$ Département des Sciences de la Terre, Faculté des Sciences, Université de Douala, Douala, Cameroon \\ ${ }^{2}$ Département de Séismologie et Géologie, Observatoire volcanologique de Goma-RDC, Goma, République Démocratique du \\ Congo (RDC) \\ ${ }^{3}$ Département des Sciences de la Terre, Faculté des Sciences, Université de Yaoundé I, Yaoundé, Cameroon \\ ${ }^{4}$ Laboratoire de Géologie de l'Environnement (LGE), Faculté des Sciences, Université de Dschang, Dschang, Cameroon \\ ${ }^{5}$ Université de Brest (UBO), Domaines Océaniques, Institut Universitaire Européen de la Mer, Place Copernic, Plouzané, \\ France \\ Email: *tchuimegniengongang@yahoo.fr, *njombiepm@yahoo.fr, francois_mvondo@hotmail.com, ibadriyo@gmail.com, \\ essombaphilippe887@yahoo.fr, Nathalielove34@yahoo.fr, diyoumen@yahoo.fr,pkamgan@yahoo.fr, Gilles.Chazot@univ-brest.fr
}

How to cite this paper: Tchuimegnie Ngongang, N.B., Wagsong, M.P.N., Owono, F.M., Badriyo, I., Essomba, P., Tchikankou, N.L.N., Youmen, D., Kamgang, P. and Chazot, G. (2021) Mineralogy and Magmatic Processes of Cenozoic Intraplate Alkaline Volcanic Rocks of Bafang and Its Environs (Cameroon Volcanic Line, West Africa). Open Journal of Geology, 11, 210-238.

https://doi.org/10.4236/ojg.2021.116013

Received: May 4, 2021

Accepted: June 21, 2021

Published: June 24, 2021

Copyright () 2021 by author(s) and Scientific Research Publishing Inc. This work is licensed under the Creative Commons Attribution International License (CC BY 4.0).

http://creativecommons.org/licenses/by/4.0/ (c) (i) Open Access

\begin{abstract}
Alkaline basalts of Bafang and its environs are consisted of feldspars, olivines, pyroxenes and oxides which appear as phenocrysts, microphenocrysts and microcrysts. Feldspars are plagioclases $\left(\mathrm{An}_{67.97-15.84} \mathrm{Ab}_{69.19-30.43} \mathrm{Or}_{20.59-1.51}\right)$ and anorthoclases $\left(\mathrm{Ab}_{68.11-61.20} \mathrm{Or}_{33.87-20.91} \mathrm{An}_{10.98-4.93}\right)$. Plagioclases are the most abundant amount these feldspars. Anorthoclases appear only in mugearite (BAF 3 and BAF 37) the most differentiated of the studied alkaline-basalts. In High Magnesian basalt, (HMg-B) plagioclases are labradorites $\left(\mathrm{An}_{67.97-59.30} \mathrm{Ab}_{38.74-30.43} \mathrm{Or}_{\text {2.75-1.60 }}\right)$ and sanidine $\left(\mathrm{An}_{45.44-31.82} \mathrm{Ab}_{62.66-51.79} \mathrm{Or}_{5.52-2.77}\right)$, whereas in Low Magnesian basalt (LMg-B) there are labrador $\left(\mathrm{An}_{67.4 .75-51.96} \mathrm{Ab}_{44.98-33.72} \mathrm{Or}_{3.06-1.51}\right)$, andesine $\left(\mathrm{An}_{45.44-31.82} \mathrm{Ab}_{62.66-51.79} \mathrm{Or}_{5.52-2.77}\right)$, oligoclase $\left(\mathrm{An}_{26.65-15.84} \mathrm{Ab}_{69.19-63.57} \mathrm{Or}_{\text {20.59-8.55 }}\right)$ and anarthoclase $\left(\mathrm{Ab}_{68.11-61.20} \mathrm{Or}_{33.87-20.91} \mathrm{An}_{10.98-4.93}\right)$. Olivines are magnesian $\left(\mathrm{Fo}_{86.7-50.1}\right)$ and ferriferous $\left(\mathrm{Fo}_{48.8-37.8}\right)$. In $\mathrm{HMg}-\mathrm{B}$, olivine are only magnesian. These olivines are chrysolites and hyalositerite. In $\mathrm{LMg}-\mathrm{B}$, olivines are magnesian and ferriferous with the predominance of ferriferous. They are chrysolites, hyalositerite and hortonolite. Pyroxenes are $\mathrm{Ca}, \mathrm{Mg}$ and $\mathrm{Fe}$ clinopyroxenes. There are diopsides $\left(\mathrm{Wo}_{51.94-45.02} \mathrm{En}_{44.41-33.16} \mathrm{Fs}_{16.42-10.70}\right)$ and augites

$\left(\mathrm{Wo}_{44.88-43.64} \mathrm{En}_{41.03-37.04} \mathrm{Fs}_{18.25-14.43}\right)$. Oxides are magnetites represented by ulvos-
\end{abstract}


pinel $\left(\mathrm{Usp}_{90-75} \mathrm{Sp}_{2-7} \mathrm{Mt}_{5-23}\right)$. Fractionation of ferromagnesian minerals (opaque oxide, olivine and pyroxene) is the main differentiation process. Two stages of fractional crystallization can be distinguished: the first stage comes with basanites and the second with hawaiites to mugearites. Chemical compositions of phenocrystals in studied basaltics lavas record signatures of magma recharge by pulsatory intrusions of new magma into the existing magma reservoir before the eruptions.

\section{Keywords}

Cameroon Volcanic Line, Bafang, Alkaline Basaltic Lavas, Fractional Crystallization, Magma Recharge

\section{Introduction}

The generation and the evolution of magma are known to be two stages related to the formation of igneous rocks. The Cameroon Volcanic Line (Figure 1(A)) is the unique tectonomagmatic feature all over the world [1] which the origin is still debated. Mineralogical studies of basaltic rocks provide informations for the better understanding of petrogenitic processes developed in the Cameroon Volcanic Line (CVL). Here we present mineral chemistry of alkaline volcanic rocks of Bafang and its environs. These volcanic rocks are basanites, alkali basalts, hawaiites and mugearites [2]. According to [2], the basaltic lavas of Bafang and its environ are alkaline volcanic rocks with $\mathrm{Na}\left(\mathrm{Na}_{2} \mathrm{O} / \mathrm{K}_{2} \mathrm{O}=1.5-3.0\right)$ and also $\mathrm{Ne}-\mathrm{Ol}-\mathrm{Di}-\mathrm{Hy}$ normative. According to $\% \mathrm{MgO}$ and their mantle sources, these basaltic rocks are subdivided into two groups: the high $\mathrm{MgO}$ basalts $(\mathrm{HMg}-\mathrm{B})$ and the low MgO basalts (LMg-B). The HMg-B is made of samples BAF 22, BAF 34, BAF 42, BAF 42 and BAF 44 with $\mathrm{MgO}$ (9.12 wt $\%$ - $11.81 \mathrm{wt} \%$ ) and the $\mathrm{LMg}-\mathrm{B}$ made of samples BAF 2, BAF 3, BAF 5a, BAF 10, BAF 11, BAF 13, BAF 15, BAF 18, BAF 33, BAF 36, BAF 37, BAF 38, BAF 40, BAF 41, BAF 43 and BAF 46 with $\mathrm{MgO}(2.38$ wt $\%-6.69 \mathrm{wt} \%)$. Mineral chemistry is used in this study to give new constraints into magmatic processes beneath the volcanic region of Bafang and its environs.

\section{Geological Setting}

The basaltic lavas from Bafang and its environs (Figure 1(B)) belong to the Bamileke plateau located in the West Cameroon Highlands (Figure 2). The study erea is $5^{\circ} 4^{\prime} 21^{\prime \prime}$ to $5^{\circ} 13^{\prime} 38^{\prime \prime}$ North and $10^{\circ} 8^{\prime} 17^{\prime \prime}$ to $10^{\circ} 19^{\prime} 38^{\prime \prime}$ East and covers surface of $500 \mathrm{~km}^{2}$ for the culminant point at $1300 \mathrm{~m}$ high (Figure $1(B)$ ). It is bounded to the north by Monts Bambouto [6], Bamenda ([7] [8] [9] [10]) and Oku ([11] [12]), to the south-west by Manengouba Mont ([13]), to the east by Monts Bana ([14] [15] [16]) and Bangou ([17]) and to the west by Mbo Plaines (Figure 2). The basaltic lavas of Bafang and its environs cover granito-gnessitic basement rocks ([14] [15] [18]) and poured out during three volcanic eruptions in the upper Miocene (10 to $6 \mathrm{Ma}$ [2] [19]). 


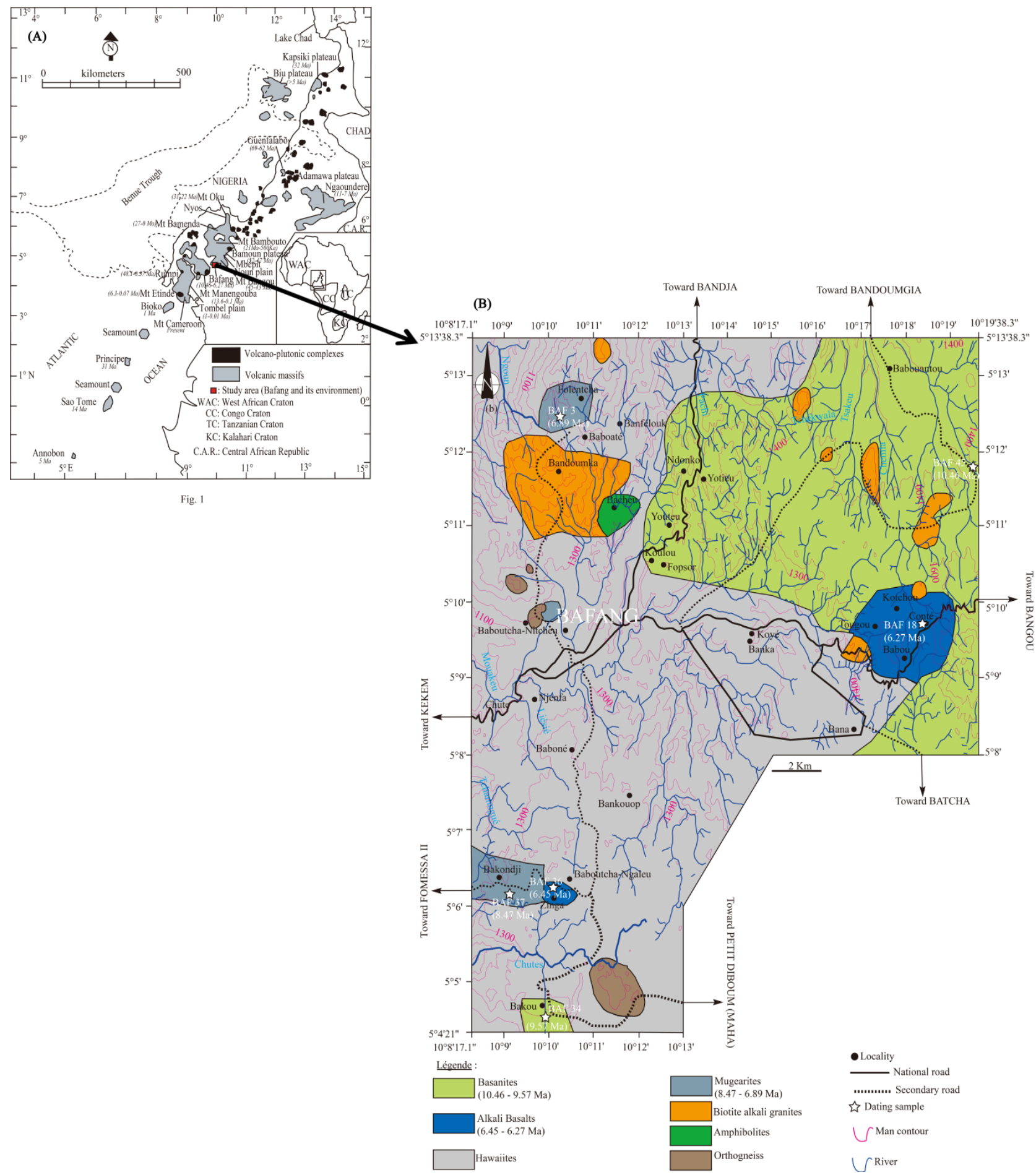

Figure 1. Study area. (A) Location of the Bafang area (Red square) along the Cameroon Volcanic Line (adapted after [3], completed). Location of seamounts after [4]. Inset, bottom right is after [5]. (B) Geological map of Bafang and its environs.

\section{Analytical Methods}

\subsection{Major and Trace Element Analyses}

Whole-rock major elements were measured on the Horiba Jobin-Yvon Ultima 2 


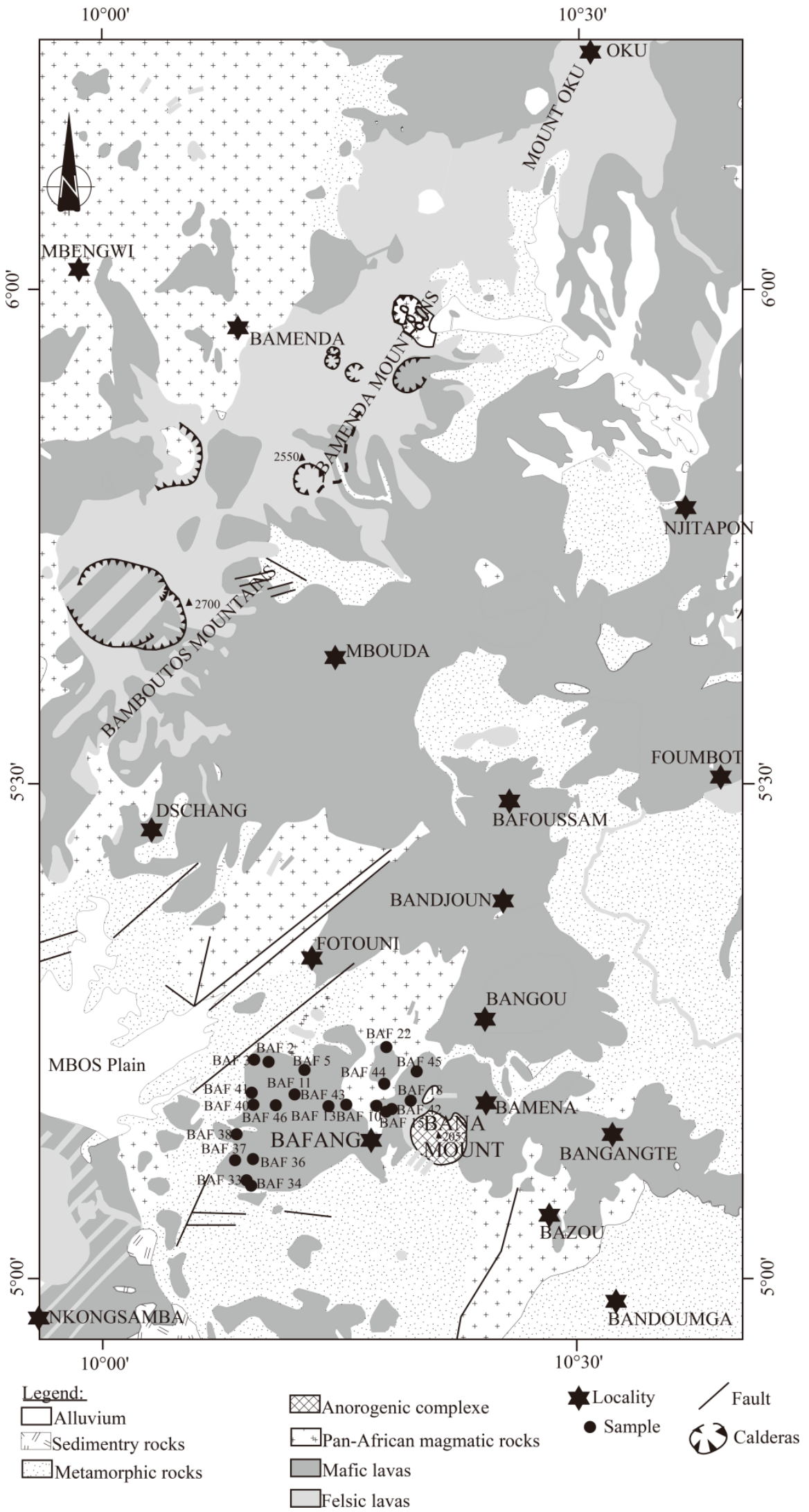

Figure 2. Simplified geological map of the studied area and location of the samples in the West Cameroon Highlands (after [20], modified). 
ICP-AES at the IUEM (European Institute for Marine Studies, Pôle de Spectrométrie Océan, Brest, France). The detailed description of the analytical procedure is given in [21]. Major elements were determined from an $\mathrm{H}_{3} \mathrm{BO}_{3}$ solution, boron being used as internal standard for ICP-AES analysis. For major elements, relative standard deviation was $1 \%$ for $\mathrm{SiO}_{2}$ and $2 \%$ for the other major elements, except for low values $(<0.50 \mathrm{wt} \%)$, for which the absolute standard deviation is $\pm 0.01 \mathrm{wt} \%$. Trace element concentrations were measured with a Thermo Element2 HR-ICP-MS in Brest (France), after a repeated $\mathrm{HF}^{-} \mathrm{HClO}_{4}$ digestion, and $\mathrm{HNO}_{3}$ dilutions (see [22] for details). The repeated analysis of the international standard BCR2 demonstrated an external reproducibility better than $5 \%-10 \%$ depending on the element and concentration.

\subsection{Minerals Analysis}

The polished thin sections of the different rock-types were observed under an Olympus BH2-HLSH microscope. The minerals of the different thin sections were observed at magnifications $\times 5, \times 10, \times 25$ and $\times 50$. Microprobe data of basaltic lavas of Bafang and its environs of Bafang were obtained using a Cameca SX-100 automated electron microprobe at Service Microsonde Ouest of Brest, France. Chemical analyzes ("Université de Bretagne Occidentale-Brest, France") of major elements were performed using an electron microprobe with beams of: 10 and $40 \mu \mathrm{m}, 15 \mathrm{kV}$ accelerating potential and $10-12 \mathrm{nA}$ current and $6 \mathrm{~s}$ counting time per element (see [23]) for analytical details]. Errors considered for these analyzes are between $5 \%$ and $10 \%$ of measured values $<1 \%$ and between $1 \%$ and $5 \%$ of the measured values $>1 \%$. The main mineralogical data are given in Table 1 .

\section{Results}

\subsection{Field Observations and Petrography}

The alkaline basaltic lavas of Bafang and its environs occur as flow, prismatic, bowl and bloc lavas generally in the summit and the side of the hill as well as in the water flows. Prismatic lavas are found in the localities of Kotchou and Njenfa. They are thick lavas with collonades form of $2 \mathrm{~m}$ high (Figure 3(A)) observed in some places. Prismatic lavas are hexagonal to pentagonal with 5 to $30 \mathrm{~cm}$ in diameter and deliminated by tensional cracks (Figure 3(B)). At Kotchou and Bana, prismatic lavas are exhumed horizontally in the soil and recover by one to two meter of soils. At Njenfa and Bafang, prismatic lavas dip to the south west and to the south indicating two directions of flowing (Figure 3(C) and Figure 3(D)). Oblic prismatic lavas dipping to the south west are qualified to false collonades whereas vertical prismatic lavas dipping to the south are true collonades. All the basaltic lavas from Bafang and its environ are mesocrate, melanocrate to holomelanocrate with phorphyritic to aphyritic and sometime fluidal textures (Figure 4). Some samples in thin section show mineral inclusions and zonations. Theses textural features are observed with olivines and clinopyroxene crystals exhibiting oxide (Figure $4\left(\mathrm{~A}^{\prime}\right)$ ) and olivines inclusions respectively. Zonations 

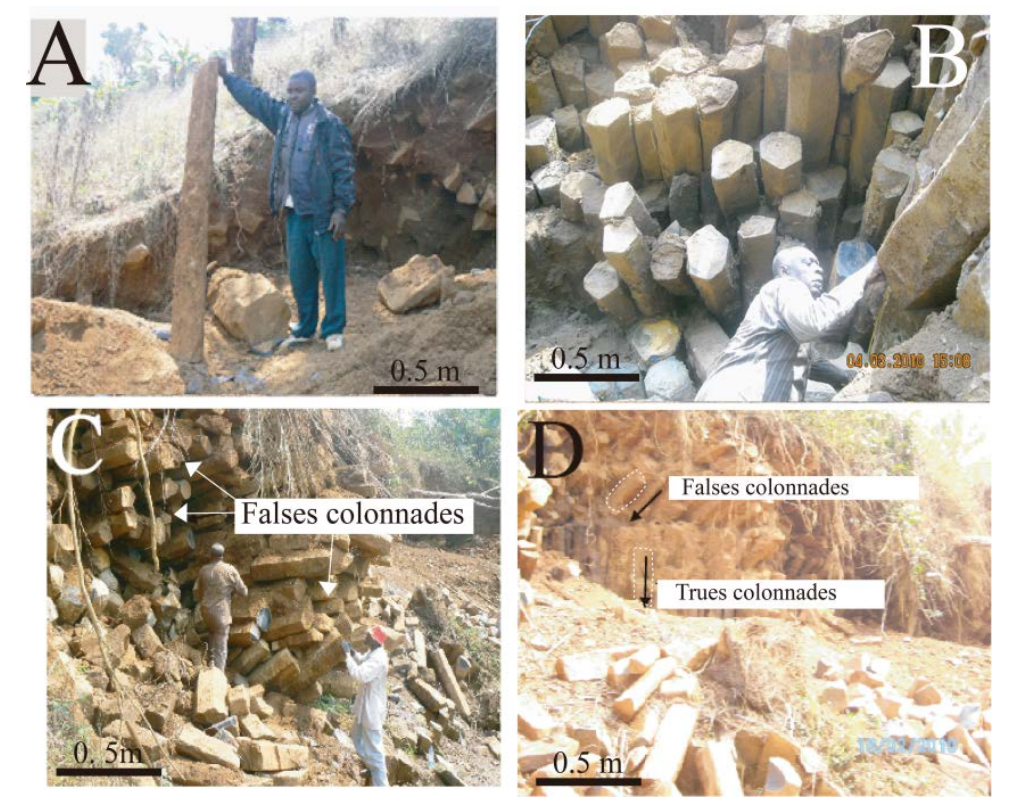

Figure 3. Prism and different colonnades in the craft quarrying of Kotchou and Njenfa. (A) colonnades of olivine and pyroxene basalts in the craft quarrying of Kotchou (N05 09'55.9", E10 17'46.8" $1610 \pm 3 \mathrm{~m}$ ), showing prisms with many faces and buried in the soil; (B) colonnades of olivine and pyroxene basalt in the craft quarrying of Njenfa (N0508'33.1", E10 09'38.6" $1197 \pm 3 \mathrm{~m}$ ), showing different types of prisms with many faces (trigonal, pentagonal and hexagonal) and more than $2 \mathrm{~m}$ length; (C) disposition of olivine and pyroxene basalt false collonnades in the craft quarryinf Njenfa; (D) disposition of olivine and pyroxene basalt true (vertical arrow) and false (oblic arrow) colonnades in the craft quarryinf Njenfa. Arrow indicate the disposition of colonnades whereas white dashed line indicate the length.

Table 1. Main mineralogical characteristics for alkaline lavas of Bafang and its environs. $\varphi=$ phenocryst; $\mu \varphi=$ microphenocryst; $\mu$ $=$ microcryst or microlite.

\begin{tabular}{ccccccc}
\hline Alkaline lavas of Bafang and its environs & Position & Olivine & Clinopyroxene & Feldspar & Fe-Ti oxide \\
\hline High MgO basalt (HMg-B) & $\varphi$ & $\mathrm{Fo}_{85-75}$ & $\mathrm{Wo}_{47-44} \mathrm{En}_{41-38} \mathrm{Fs}_{18-14}$ & & & \\
& $\mu \varphi$ & & & & & \\
& $\mu$ & $\mathrm{Fo}_{74-67}$ & $\mathrm{Wo}_{45} \mathrm{En}_{38} \mathrm{Fs}_{17}$ & $\mathrm{An}_{68-48} \mathrm{Ab}_{40-39} \mathrm{Or}_{12-2}$ & $\mathrm{USP}_{90-88} \mathrm{Sp}_{7-4} \mathrm{Mt}_{8-5}$ \\
\hline Low MgO basalt (LMg-B) & $\varphi$ & $\mathrm{Fo}_{86-40}$ & $\mathrm{Wo}_{51-44} \mathrm{En}_{41-36} \mathrm{Fs}_{18-11}$ & $\mathrm{An}_{65-27} \mathrm{Ab}_{65-34} \mathrm{Or}_{9-2}$ & $\mathrm{USP}_{81-75} \mathrm{Sp}_{4-2} \mathrm{Mt}_{22-15}$ \\
& $\mu \varphi$ & $\mathrm{Fo}_{52-51}$ & & & $\mathrm{An}_{54-16} \mathrm{Ab}_{69-44} \mathrm{Or}_{14-2}$ & $\mathrm{USP}_{89-85} \mathrm{Sp}_{5-2} \mathrm{Mt}_{11-8}$ \\
& $\mu$ & $\mathrm{Fo}_{67-38}$ & $\mathrm{Wo}_{52-49} \mathrm{En}_{38-33} \mathrm{Fs}_{15-13}$ & $\mathrm{An}_{57-5} \mathrm{Ab}_{69-42} \mathrm{Or}_{34-2}$ & $\mathrm{USP}_{89-75} \mathrm{Sp}_{5-2} \mathrm{Mt}_{23-9}$ \\
\hline
\end{tabular}

are observed with olivine, clinopyroxene and plagioclase phenocrysts (Figure $4\left(C^{\prime}\right)$ ). The decrease of olivine and clinopyroxene phenocrysts is also observed from basanites to mugearites (Figure 4).

\subsection{Mineralogy}

Seven (07) thin sections of representative samples (Two HMg-B: BAF 22 and BAF 42 and five LMg-B: BAF 2, BAF 3, BAF 18, BAF 36 and BAF 37) have been chosen for microprobe analysis in order to determine chemical compositions of 

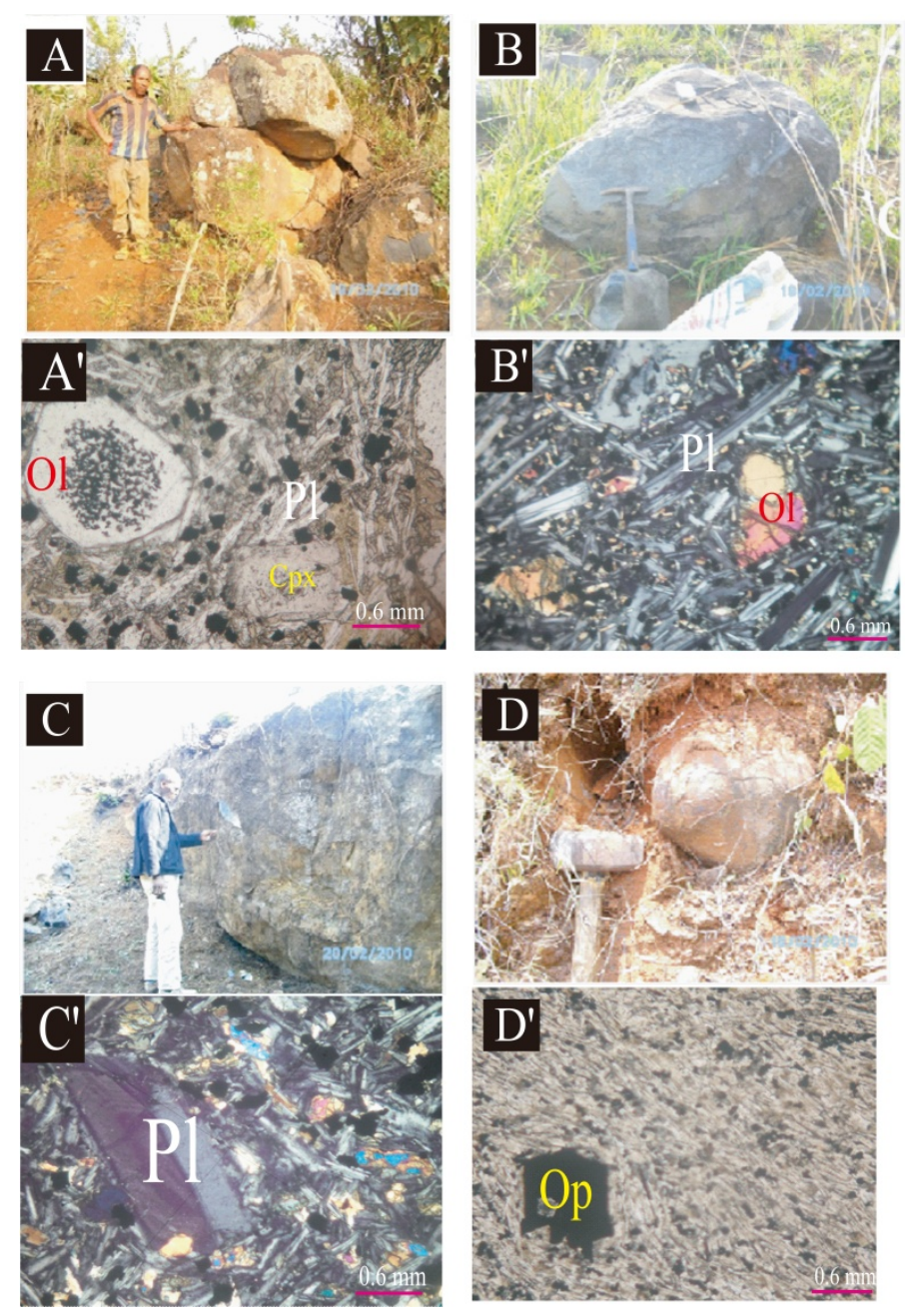

Figure 4. Photomicrographs and microscopic images of representative samples of alkaline basaltic lavas from Bafang and its environs. (A) and (B) Fields observation of HMg-B; (C) and (D) Fields observation of LMg-B. (A') and (B') microscopic images of microlitic porphyric texture of HMg-B. $\left(C^{\prime}\right)$ and $\left(D^{\prime}\right)$ microscopic images of microlitic porphyric and aphyric textures of LMg-B. These observations are in cross and under-polarized light. Pl: plagioclase; Cpx: clinopyroxene; Ol: olivine; Op: Opaque mineral.

different mineral phases. One hundred and eight chemical analyses have been performed on phenocrysts, microphenocrysts and microcrysts of olivine, clinopyroxene, oxide, plagioclase and anorthoclase in these samples (Tables 2-5). In these seven thin sections of samples analysed one is from basanite, three from alkali basalt, one from hawaiite and two from mugearite.

\subsubsection{Olivine}

Olivine crystals analysed (Table 2) are characterized by their higher content in $\mathrm{MgO}$ relative to $\mathrm{FeO}$, synonymous with their forsterite composition $\left(\mathrm{Fo}_{86-75}\right)$ in $\mathrm{HMg}-\mathrm{B}$. But in LMg-B, olivines in hawaiite and mugearite have higher content in $\mathrm{FeO}$ relative to $\mathrm{MgO}$, synonymous with their fayalite composition $\left(\mathrm{Fo}_{38-53}\right)$. The range of olivine composition is shown in Figure 5. In this Figure 5, olivines of $\mathrm{HMg}-\mathrm{B}$ are chrysolite and hyalosiderite; and those of $\mathrm{LMg}-\mathrm{B}$ are chrysolite, 


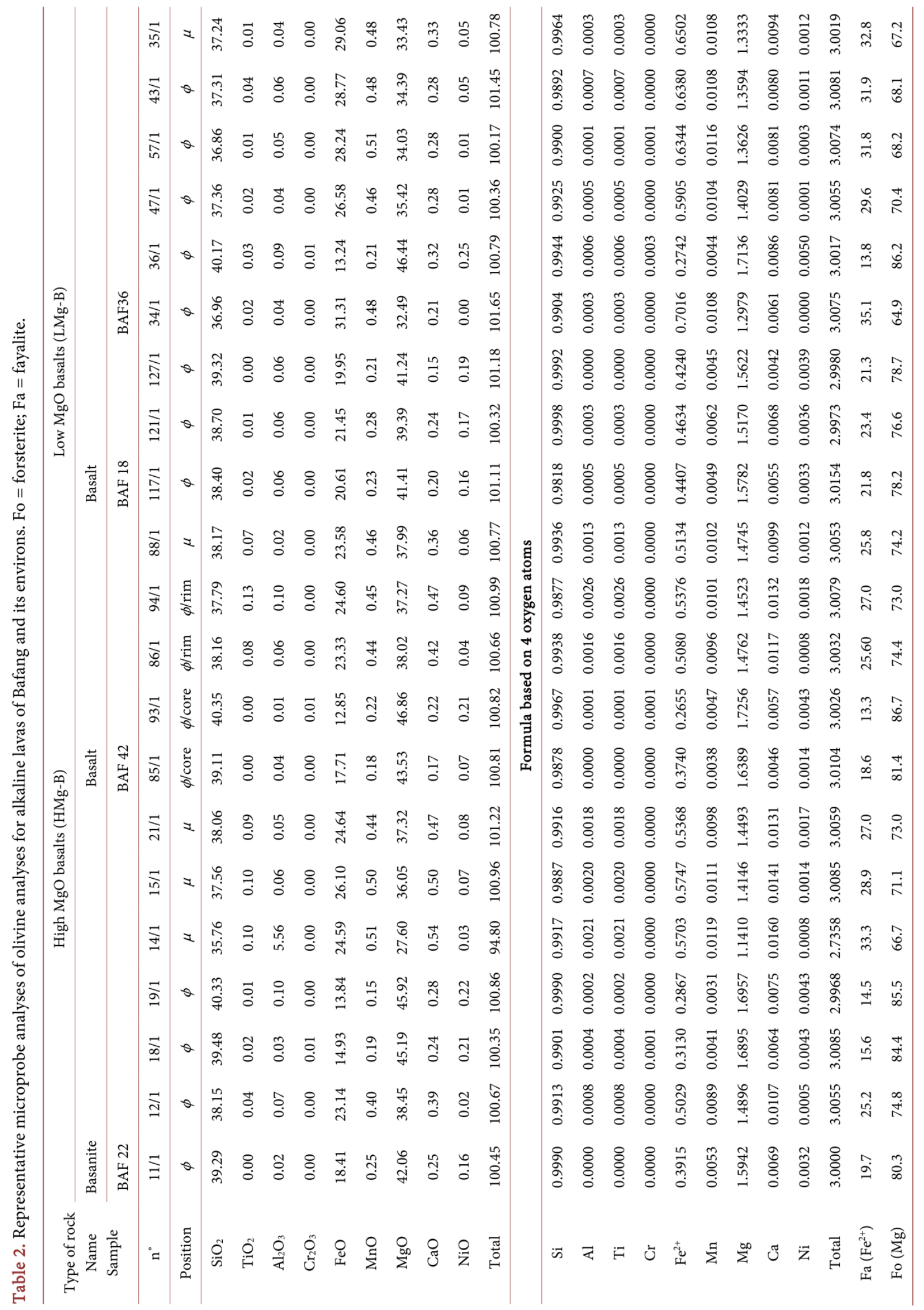




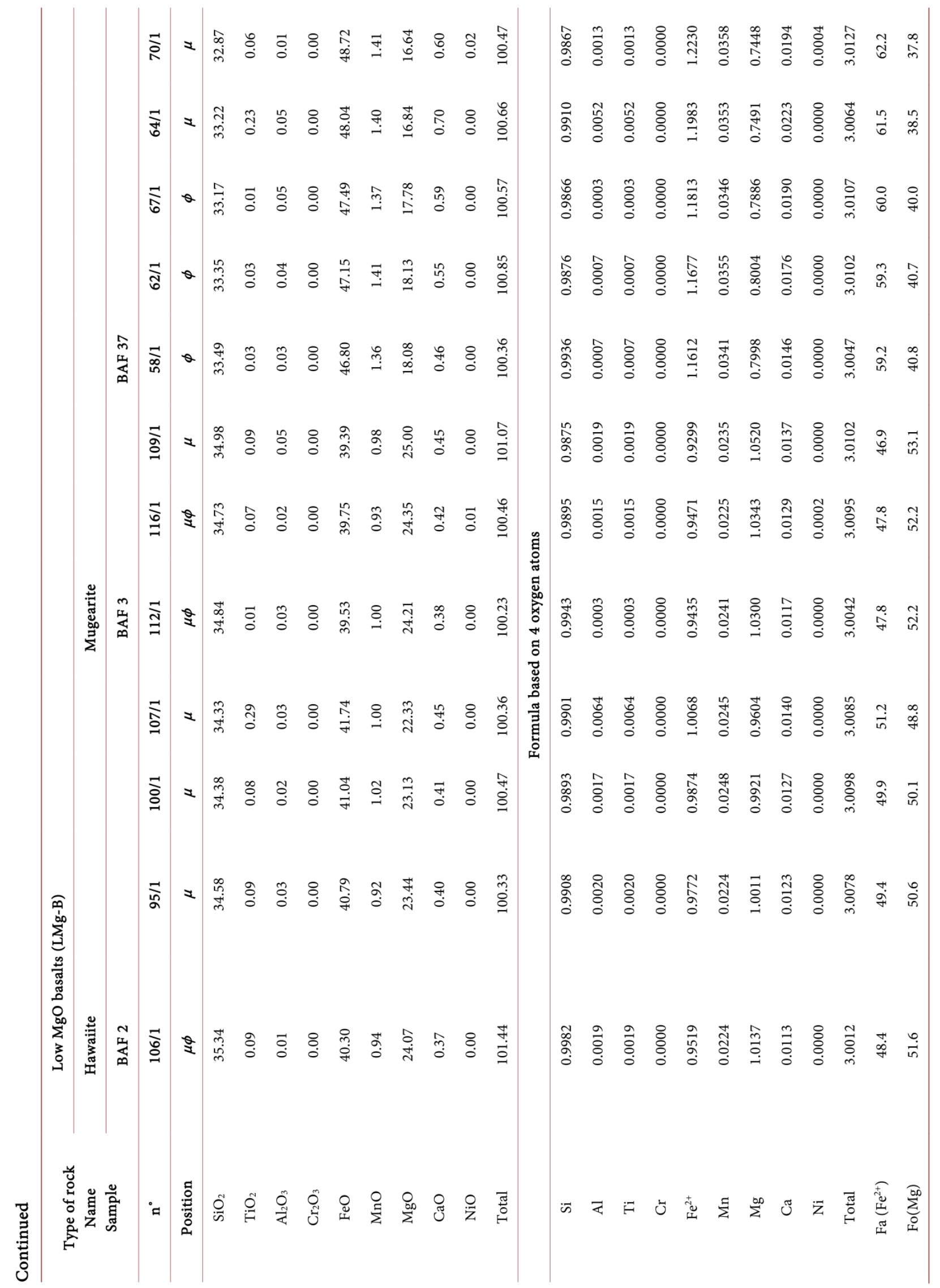




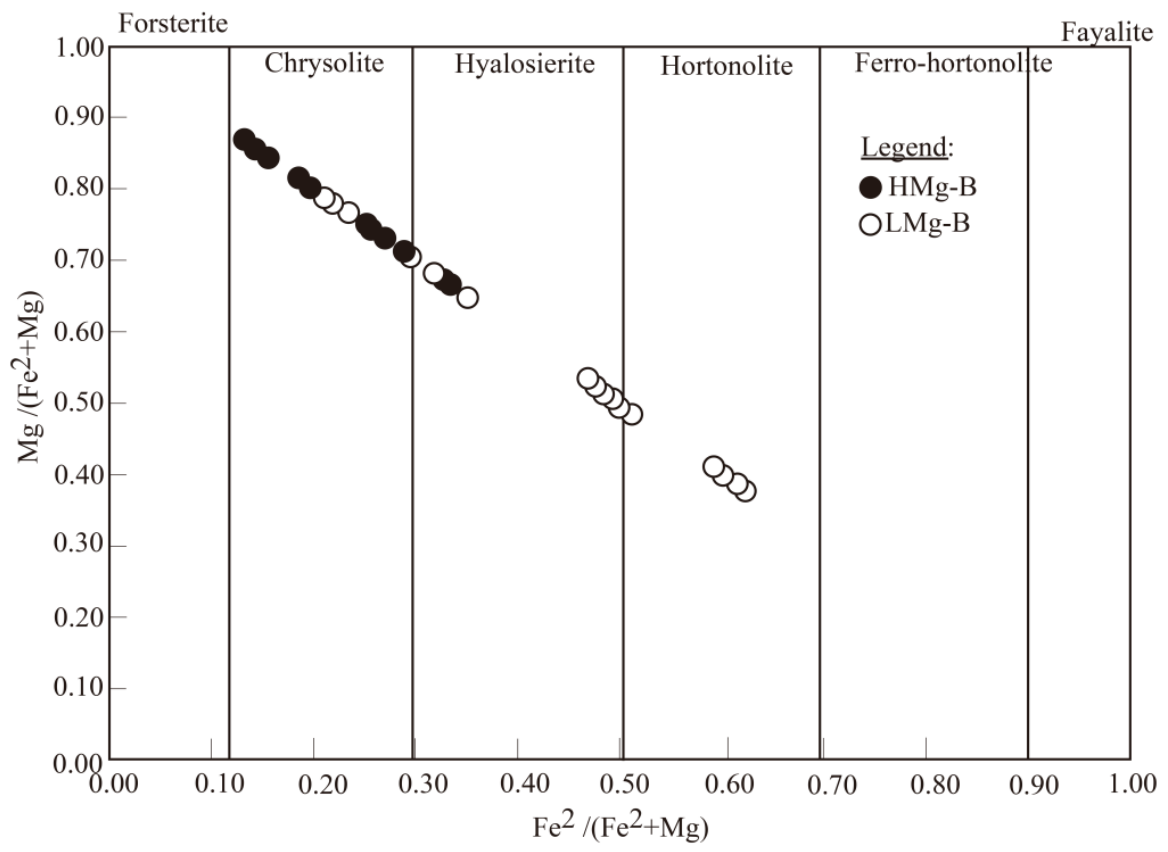

Figure 5. End-member compositional variation in olivine of alkaline basaltic lavas from Bafang and its environs.

hyalosiderite and hortonolite. Olivine compositions in $\mathrm{HMg}-\mathrm{B}$ range from $\mathrm{Fo}_{67}$ to $\mathrm{Fo}_{87}$ with $0.17 \mathrm{wt} \%-0.54 \mathrm{wt} \% \mathrm{CaO}$ and $0.15 \mathrm{wt} \%-0.51 \mathrm{wt} \% \mathrm{MnO}$. In the $\mathrm{LMg}-\mathrm{B}$, olivines composition range from $\mathrm{Fo}_{38}$ to $\mathrm{Fo}_{86}$ with $0.15 \mathrm{wt} \%-0.70 \mathrm{wt} \%$ $\mathrm{CaO}$ and $0.18 \mathrm{wt} \%-1.41 \mathrm{wt} \% \mathrm{MnO}$. In $\mathrm{HMg}-\mathrm{B} \mathrm{CaO}$ shows negative correlation while $\mathrm{NiO}$ display positive correlation (Figure 6). $\mathrm{CaO}$ in olivine phenocrysts display positive correlation in core and negative in rim contrary to $\mathrm{NiO}$ (Figure 6(E) and Figure 6(F)) whereas in $\mathrm{LMg}-\mathrm{B} \mathrm{CaO}$ and $\mathrm{NiO}$ variations with Fo are contrasted (Figure 7). $\mathrm{CaO}$ and $\mathrm{NiO}$ contents in the studied basaltic lavas are in the range generally obtained in alkali basalts from the Cameroon volcanic Line ([1] [10] [24] [25] [26]). In these rocks, olivine phenocrysts are generally more magnesian and exhibit zoning, with $\mathrm{Fe}$ enrichment from core $\left(\mathrm{Fo}_{81-87}\right)$ to rim $\left(\mathrm{Fo}_{73-74}\right)$, suggesting equilibrium at $\sim 1150^{\circ} \mathrm{C}-1200^{\circ} \mathrm{C}([27][28])$.

\subsubsection{Clinopyroxene}

Structural formular have been calculated according to [29]. Results are shown in Table 3. In the binary plot Q-J $\left(\mathrm{Q}=\mathrm{Ca}+\mathrm{Mg}+\mathrm{Fe}^{2+}\right.$; J = 2Na) of [29] (Figure $8(\mathrm{~A})$ ), pyroxenes in the studied mafic lavas in the area of Bafang fall in the Quad ( $\mathrm{Ca}-\mathrm{Mg}-\mathrm{Fe}$ ) domain. They are $\mathrm{Ca}-\mathrm{Mg}-\mathrm{Fe}$ pyroxenes regarding thier relative contents of $\mathrm{Ca}_{2} \mathrm{Si}_{2} \mathrm{O}_{6}$ (Wo), $\mathrm{Mg}_{2} \mathrm{Si}_{2} \mathrm{O}_{6}$ (En) et $\mathrm{Fe}_{2}{ }^{2+} \mathrm{Si}_{2} \mathrm{O}_{6}$ (Fs). In the ternary plot (Wo-En-Fs; Figure 8(B)) of [29], these pyroxenes are clinopyroxenes and mainly augite $\left(\mathrm{Wo}_{44.9-43.6} \mathrm{En}_{41.0-37.0} \mathrm{Fs}_{18.3-14.4}\right)$ and diopside $\left(\mathrm{Wo}_{51.9-45.0} \mathrm{En}_{41.4-33.2} \mathrm{Fs}_{16.4-10.7}\right)$ in compositions.

Structural formula indicates Si permanent gap in the tetraedric site $\mathrm{T}$ and the presence of $85 \%$ of ferriferous iron in the octaedric sites. Oxides in the clinopyroxene in the erea of Bafang are $\mathrm{TiO}_{2}\left(0.3\right.$ à 5.4\%wt), $\mathrm{Al}_{2} \mathrm{O}_{3}(1.0-9.6 \% w t), \mathrm{Na}_{2} \mathrm{O}$ 

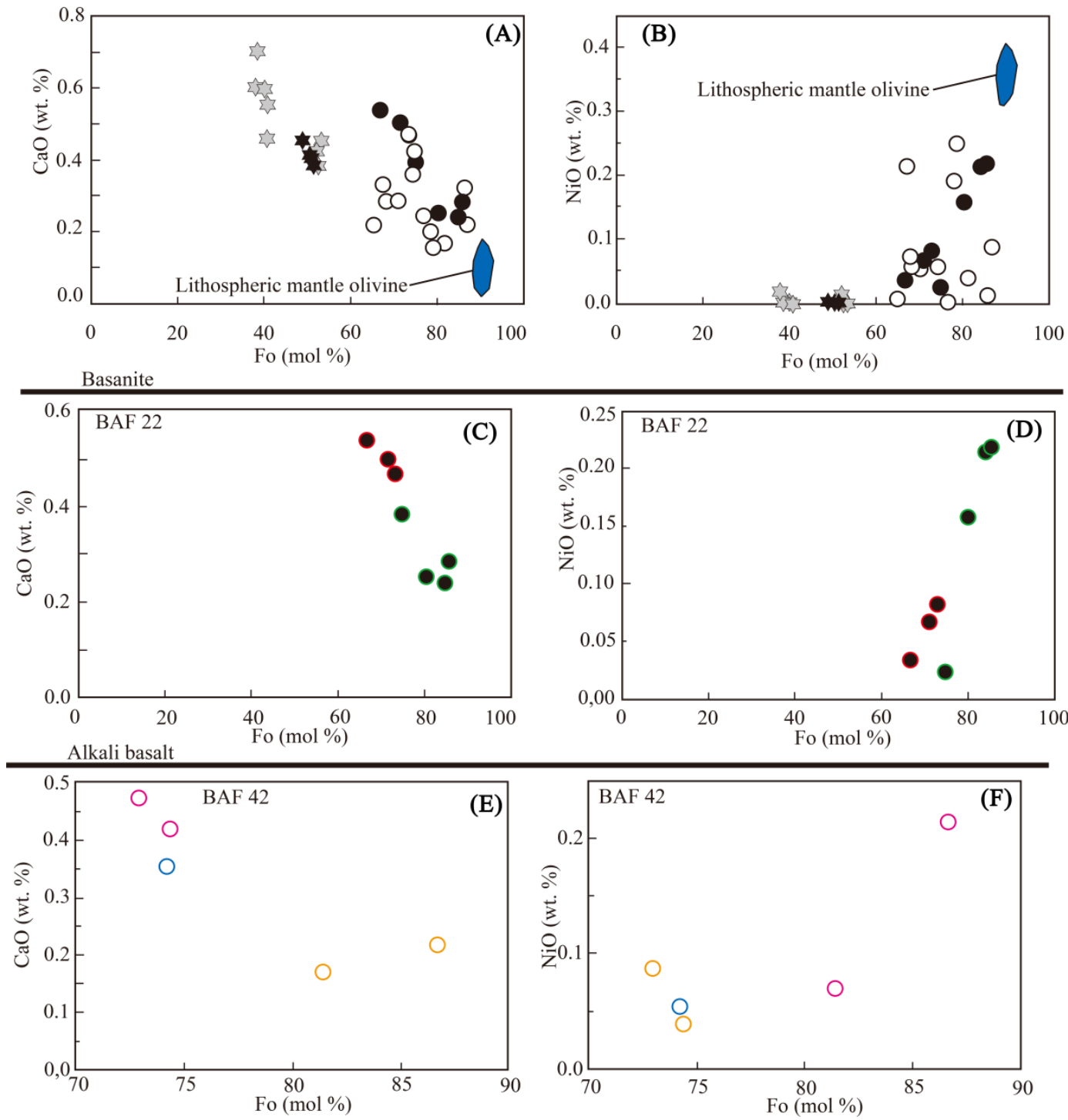

Figure 6. Relations between $\mathrm{Fo}$ and $\mathrm{CaO}$, and between $\mathrm{Fo}$ and $\mathrm{NiO}$ of olivines in basanites and alkali basalt of HMg-B of Bafang. Black circle (basanites), White circle (alkali basalt), Green (phenocryst), Red (micro-phenocryst), Blue (microcryst), Orange (core), Pink (rim).

(0.4 - 1.8\%wt), $\mathrm{FeO}(5.7-14.2 \% \mathrm{wt}), \mathrm{MgO}$ (10.2 - 14.6\%wt), $\mathrm{CaO}$ (19.8 23.3\%wt), $\mathrm{Cr}_{2} \mathrm{O}_{3}(0-0.66 \% w t)$ and $\mathrm{MnO}(0.1-0.5 \% w t)$.

Chemical compositions of the representative clinopyroxene are shown in a binary and ternary plot of the Wo-En-Fs system (Table 3; Figure 8). According to binary plot (Figure $8(\mathrm{~A})$ ), pyroxene of Bafang are $\mathrm{Ca}-\mathrm{Mg}$-Fe rich. In ternary plot (Figure 8(B)), pyroxene in alkaline volcanic rocks of Bafang are clinopyroxene and have compositions ranging from diopside $\left(\mathrm{Wo}_{51.9-45.0} \mathrm{En}_{41.4-33.2} \mathrm{Fs}_{16.4-10.7}\right)$ to augite $\left(\mathrm{Wo}_{44.9-43.6} \mathrm{En}_{41.0-37.0} \mathrm{Fs}_{18.3-14.4}\right)$. Diopsides are more abundant than augites. But in basanite, augites are predominent.

Clinopyroxene in $\mathrm{HMg}-\mathrm{B}$ range from $\mathrm{Wo}_{51.2} \mathrm{En}_{41.0} \mathrm{Fs}_{18.3}$ to $\mathrm{Wo}_{43.8} \mathrm{En}_{35.5} \mathrm{Fs}_{11.9}$. $\mathrm{TiO}_{2}(0.31-5.39 \mathrm{wt} \%), \mathrm{Al}_{2} \mathrm{O}_{3}(2.55-8.29 \mathrm{wt} \%), \mathrm{Na}_{2} \mathrm{O}(0.42-1.79 \mathrm{wt} \%)$ and $\mathrm{CaO}$ (19.76 - $23.45 \mathrm{wt} \%)$ abundances are fairly high and various indicating the alkali 
Alkali basalt
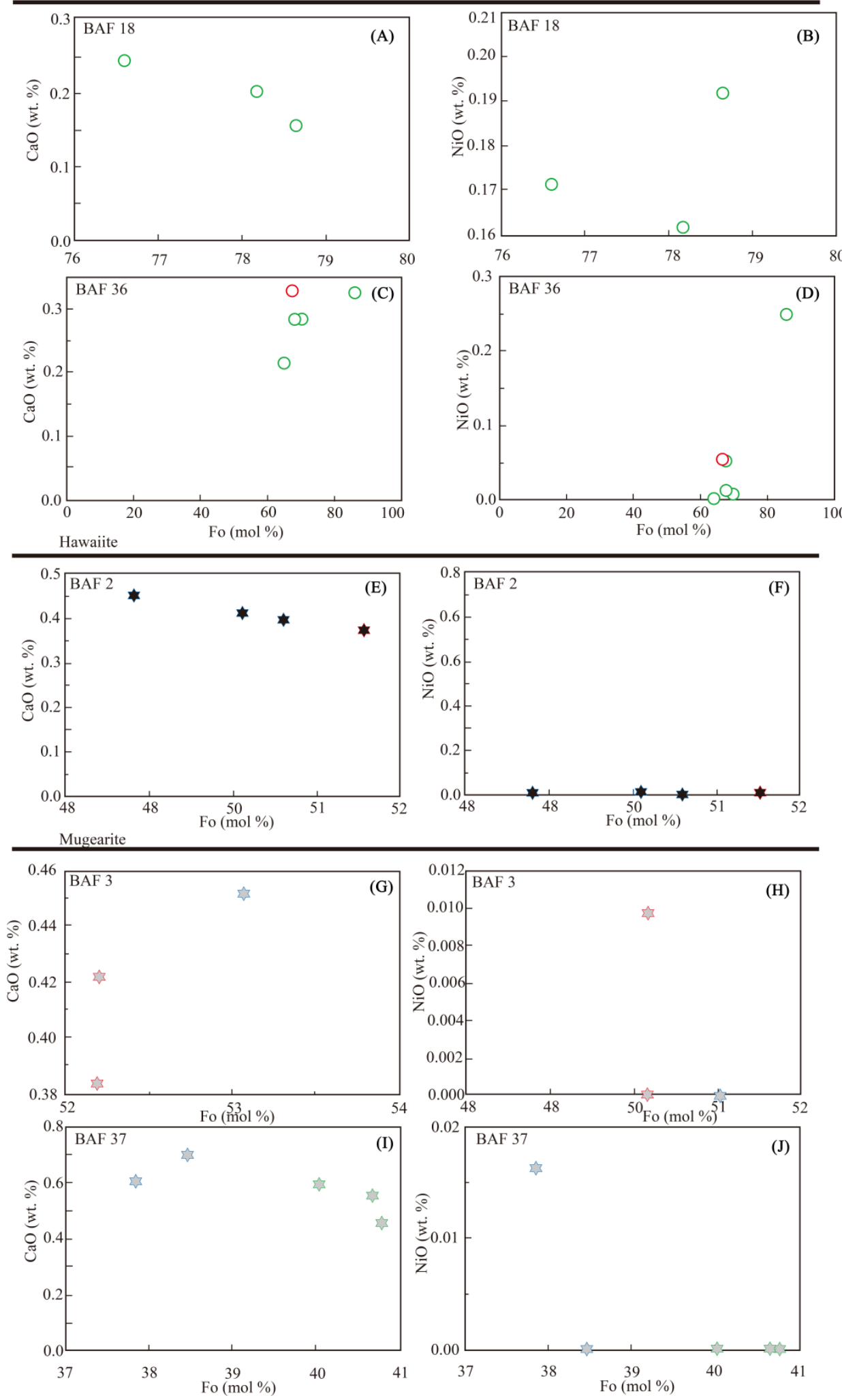

Figure 7. Relations between $\mathrm{Fo}$ and $\mathrm{CaO}$, and between $\mathrm{Fo}$ and $\mathrm{NiO}$ of olivines in alkali basalts, hawaiites and mugearites of LMg-B of Bafang and its environs. White circle (alkali basalt), Black star (hawaiites), Grey circle (mugearite), Green (phenocryst), Red (micro-phenocryst), Blue (microcryst), Pink (rim). 

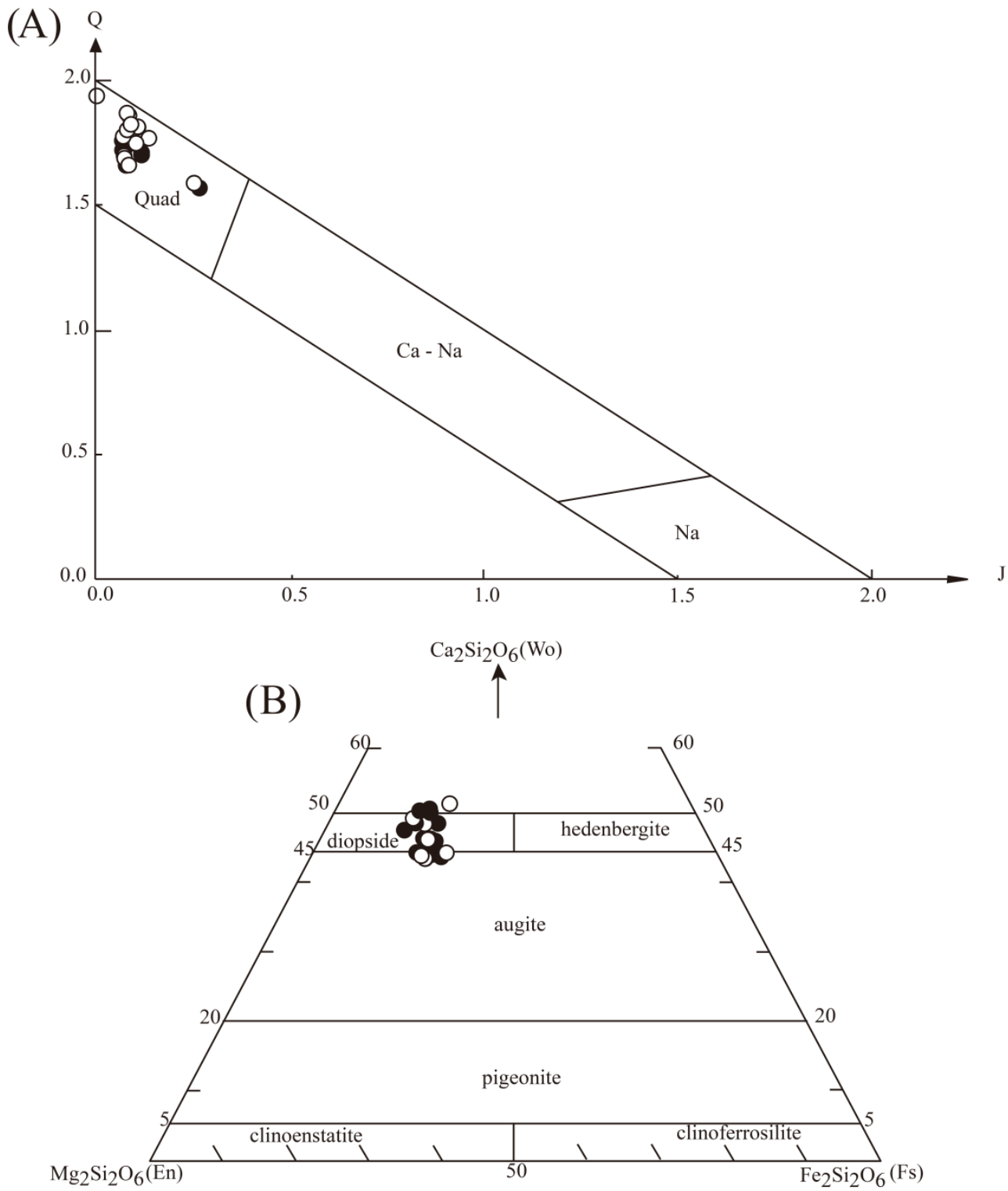

Figure 8. Nature and nomenclature of clinopyroxenes in the alkaline basaltic lavas of Bafang and its environs. (A) Position of clinopyroxenes of alkaline basaltic lavas of Bafang in the Q-J diagramme of [29]. (B) Position of clinopyroxenes of alkaline basaltic lavas of Bafang in the Wo-En-Fs diagramme of [29]. Symbols as in Figure 5.

nature of these pyroxenes [31]. Clinopyroxenes in some samples show both concentric and zoning. Zoning Cpx phenocrysts show differences in $\mathrm{TiO}_{2}, \mathrm{Al}_{2} \mathrm{O}_{3}$, $\mathrm{FeO}$ and $\mathrm{MgO}$ contents in different sectors (Table 3). Some of the larger phenocrysts are $\mathrm{Na}_{2} \mathrm{O}$ and $\mathrm{FeO}$ rich but low in $\mathrm{Al}_{2} \mathrm{O}_{3}, \mathrm{MgO}$ and $\mathrm{TiO}_{2}$ (Table 3). $\mathrm{Al} / \mathrm{Ti}$ ratios are low in the rim (2.78 - 3.49) and high in the core (4.99 - 13.10).

Cpx in LMg-B range from $\mathrm{Wo}_{51.9} \mathrm{En}_{40.7} \mathrm{Fs}_{15.7}$ to $\mathrm{Wo}_{43.6} \mathrm{En}_{33.2} \mathrm{Fs}_{12.6} . \mathrm{TiO}_{2}(0.53$ wt $\%$ - $3.25 \mathrm{wt} \%), \mathrm{Al}_{2} \mathrm{O}_{3}$ (0.97 wt $\left.\%-4.89 \mathrm{wt} \%\right), \mathrm{Na}_{2} \mathrm{O}(0.53 \mathrm{wt} \%-0.72 \mathrm{wt} \%)$ and $\mathrm{CaO}$ (21.77 wt\% - $21.96 \mathrm{wt} \%)$. These compositions shows that HMg-B Cpx are rich than LMg-B Cpx.

Clinopyroxenes with zoning in alkaline basaltic lavas of Bafang show two 
Table 3. Representative microprobe analyses of clinopyroxene for alkaline lavas of Bafang and its environs. Wo $=\mathrm{Ca}$ (wollastonite). $\mathrm{En}=\mathrm{Mg}$ (enstatite). $\mathrm{Fs}=\mathrm{Fe}^{2}+\mathrm{Fe}^{3}+\mathrm{Mn}$ (ferrosilite). $\mathrm{Mg} \#=100^{\star} \mathrm{Mg} /\left(\mathrm{Mg}+\mathrm{Fe}^{2+}\right)$, where $\mathrm{Fe}^{2+}$ is calculated after [30].

\begin{tabular}{|c|c|c|c|c|c|c|c|c|c|c|c|c|c|c|c|}
\hline \multirow{4}{*}{$\begin{array}{c}\text { Type of rock } \\
\text { Name } \\
\text { sample } \\
\mathbf{n}^{\bullet}\end{array}$} & \multicolumn{15}{|c|}{ High Magnesian-basalts (HMg-B) } \\
\hline & \multicolumn{9}{|l|}{ Basanite } & \multicolumn{6}{|l|}{ Basalt } \\
\hline & \multicolumn{9}{|c|}{ BAF 22} & \multicolumn{6}{|c|}{ BAF 42} \\
\hline & $13 / 1$ & $20 / 1$ & $24 / 1$ & $26 / 1$ & $25 / 1$ & $27 / 1$ & $30 / 1$ & $31 / 1$ & $33 / 1$ & $80 / 1$ & $81 / 1$ & $87 / 1$ & $89 / 1$ & $90 / 1$ & $91 / 1$ \\
\hline Position & $\phi$ & $\phi$ & $\phi /$ core & $\phi /$ rimext & $\phi /$ rim & $\phi$ & $\phi /$ core & $\phi /$ rim & $\mu$ & $\phi /$ core & $\phi /$ rim & $\phi$ & $\mu$ & $\phi /$ core & $\phi /$ rim \\
\hline $\mathrm{SiO}_{2}$ & 45.58 & 48.09 & 48.93 & 46.74 & 45.62 & 47.63 & 46.22 & 47.05 & 43.13 & 50.75 & 42.11 & 48.09 & 44.45 & 48.67 & 44.27 \\
\hline $\mathrm{TiO}_{2}$ & 3.41 & 1.55 & 1.49 & 3.18 & 3.38 & 1.77 & 2.98 & 2.84 & 4.44 & 0.31 & 5.39 & 2.49 & 3.68 & 1.46 & 3.89 \\
\hline $\mathrm{Al}_{2} \mathrm{O}_{3}$ & 7.69 & 6.39 & 7.45 & 6.25 & 7.33 & 5.65 & 6.91 & 6.32 & 7.25 & 2.55 & 9.57 & 5.34 & 7.94 & 6.65 & 8.29 \\
\hline $\mathrm{Cr}_{2} \mathrm{O}_{3}$ & 0.06 & 0.00 & 0.21 & 0.00 & 0.00 & 0.00 & 0.01 & 0.15 & 0.00 & 0.08 & 0.11 & 0.19 & 0.31 & 0.03 & 0.41 \\
\hline $\mathrm{FeO}$ & 7.36 & 11.00 & 6.87 & 7.04 & 7.46 & 9.11 & 7.28 & 7.26 & 10.58 & 12.61 & 7.61 & 6.69 & 7.17 & 9.19 & 6.54 \\
\hline $\mathrm{MnO}$ & 0.15 & 0.28 & 0.09 & 0.09 & 0.15 & 0.24 & 0.12 & 0.15 & 0.18 & 0.36 & 0.14 & 0.12 & 0.15 & 0.18 & 0.07 \\
\hline $\mathrm{MgO}$ & 12.06 & 10.30 & 14.61 & 12.85 & 12.35 & 12.05 & 12.85 & 13.28 & 11.98 & 11.90 & 10.91 & 14.03 & 12.39 & 11.26 & 12.30 \\
\hline $\mathrm{CaO}$ & 23.45 & 20.46 & 19.76 & 23.28 & 23.20 & 23.07 & 23.22 & 22.89 & 21.92 & 20.47 & 23.17 & 22.76 & 22.95 & 20.77 & 23.02 \\
\hline $\mathrm{Na}_{2} \mathrm{O}$ & 0.45 & 1.79 & 0.81 & 0.49 & 0.50 & 0.49 & 0.42 & 0.43 & 0.56 & 0.63 & 0.56 & 0.46 & 0.48 & 1.77 & 0.49 \\
\hline $\mathrm{K}_{2} \mathrm{O}$ & 0.01 & 0.00 & 0.00 & 0.00 & 0.00 & 0.00 & 0.00 & 0.00 & 0.03 & 0.00 & 0.00 & 0.00 & 0.01 & 0.01 & 0.00 \\
\hline $\mathrm{P}_{2} \mathrm{O}_{5}$ & 0.00 & 0.06 & 0.00 & 0.00 & 0.02 & 0.02 & 0.00 & 0.01 & 0.07 & 0.01 & 0.05 & 0.01 & 0.00 & 0.04 & 0.01 \\
\hline $\mathrm{NiO}$ & 0.04 & 0.00 & 0.03 & 0.00 & 0.00 & 0.00 & 0.00 & 0.00 & 0.00 & 0.00 & 0.01 & 0.06 & 0.00 & 0.00 & 0.00 \\
\hline Total & 100.25 & 99.91 & 100.24 & 99.91 & 100.00 & 100.04 & 100.02 & 100.38 & 100.15 & 99.67 & 99.62 & 100.22 & 99.53 & 100.01 & 99.29 \\
\hline \multicolumn{16}{|c|}{ Formula based on 4 cations and 6 oxygen atoms } \\
\hline $\mathrm{Si}$ & 1.70 & 1.80 & 1.79 & 1.74 & 1.70 & 1.78 & 1.72 & 1.74 & 1.62 & 1.92 & 1.59 & 1.78 & 1.66 & 1.80 & 1.66 \\
\hline $\mathrm{Al}^{\text {iv }}$ & 0.30 & 0.20 & 0.21 & 0.26 & 0.30 & 0.22 & 0.28 & 0.26 & 0.32 & 0.08 & 0.41 & 0.22 & 0.34 & 0.20 & 0.34 \\
\hline $\mathrm{Al}^{\mathrm{vi}}$ & 0.03 & 0.08 & 0.11 & 0.01 & 0.02 & 0.03 & 0.02 & 0.02 & 0.00 & 0.03 & 0.01 & 0.01 & 0.01 & 0.09 & 0.02 \\
\hline Alt & 0.34 & 0.28 & 0.32 & 0.27 & 0.32 & 0.25 & 0.30 & 0.28 & 0.32 & 0.11 & 0.42 & 0.23 & 0.35 & 0.29 & 0.37 \\
\hline $\mathrm{Ti}$ & 0.10 & 0.04 & 0.04 & 0.09 & 0.09 & 0.05 & 0.08 & 0.08 & 0.13 & 0.01 & 0.15 & 0.07 & 0.10 & 0.04 & 0.11 \\
\hline $\mathrm{Cr}$ & 0.00 & 0.00 & 0.01 & 0.00 & 0.00 & 0.00 & 0.00 & 0.00 & 0.00 & 0.00 & 0.00 & 0.01 & 0.01 & 0.00 & 0.01 \\
\hline $\mathrm{Fe}^{3+}$ & 0.11 & 0.16 & 0.07 & 0.10 & 0.13 & 0.13 & 0.13 & 0.11 & 0.23 & 0.07 & 0.13 & 0.10 & 0.14 & 0.15 & 0.12 \\
\hline $\mathrm{Fe}^{2+}$ & 0.12 & 0.18 & 0.14 & 0.11 & 0.11 & 0.16 & 0.10 & 0.11 & 0.10 & 0.32 & 0.11 & 0.10 & 0.08 & 0.14 & 0.08 \\
\hline $\mathrm{Mn}$ & 0.00 & 0.01 & 0.00 & 0.00 & 0.00 & 0.01 & 0.00 & 0.00 & 0.01 & 0.01 & 0.00 & 0.00 & 0.00 & 0.01 & 0.00 \\
\hline $\mathrm{Mg}$ & 0.67 & 0.57 & 0.80 & 0.71 & 0.69 & 0.67 & 0.71 & 0.73 & 0.67 & 0.67 & 0.61 & 0.77 & 0.69 & 0.62 & 0.69 \\
\hline $\mathrm{Ca}$ & 0.93 & 0.82 & 0.77 & 0.93 & 0.93 & 0.92 & 0.92 & 0.91 & 0.88 & 0.83 & 0.94 & 0.90 & 0.92 & 0.82 & 0.92 \\
\hline $\mathrm{Na}$ & 0.03 & 0.13 & 0.06 & 0.04 & 0.04 & 0.04 & 0.03 & 0.03 & 0.04 & 0.05 & 0.04 & 0.03 & 0.03 & 0.13 & 0.04 \\
\hline $\mathrm{K}$ & 0.00 & 0.00 & 0.00 & 0.00 & 0.00 & 0.00 & 0.00 & 0.00 & 0.00 & 0.00 & 0.00 & 0.00 & 0.00 & 0.00 & 0.00 \\
\hline Total & 4.00 & 4.00 & 4.00 & 4.00 & 4.00 & 4.00 & 4.00 & 4.00 & 4.00 & 4.00 & 4.00 & 4.00 & 4.00 & 4.00 & 4.00 \\
\hline $\mathrm{Mg} \#$ & 85.08 & 76.16 & 84.90 & 86.19 & 86.70 & 81.18 & 87.63 & 86.69 & 86.76 & 67.42 & 85.31 & 88.25 & 89.52 & 81.94 & 89.12 \\
\hline Wo & 46.55 & 44.14 & 44.88 & 44.56 & 45.60 & 46.62 & 44.69 & 43.75 & 44.82 & 49.38 & 51.19 & 44.70 & 50.22 & 50.47 & 47.89 \\
\hline En & 38.46 & 41.03 & 39.01 & 41.01 & 37.98 & 38.69 & 38.52 & 38.67 & 38.03 & 38.80 & 35.53 & 37.04 & 36.42 & 37.55 & 41.41 \\
\hline Fs & 15.00 & 14.83 & 16.11 & 14.43 & 16.42 & 14.69 & 16.79 & 17.58 & 17.15 & 11.81 & 13.28 & 18.25 & 13.35 & 11.98 & 10.70 \\
\hline
\end{tabular}




\section{Continued}

\begin{tabular}{|c|c|c|c|c|c|c|c|c|c|}
\hline \multirow{3}{*}{$\begin{array}{c}\text { Type of rock } \\
\text { Name } \\
\text { Sample }\end{array}$} & \multicolumn{9}{|c|}{ Low Magnesian-basalts (LMg-B) } \\
\hline & \multicolumn{2}{|l|}{ Basalt } & \multicolumn{2}{|l|}{ Hawaiite } & \multicolumn{3}{|c|}{ Mugearite } & \multirow[b]{3}{*}{$69 / 1$} & \multirow[b]{3}{*}{$72 / 1$} \\
\hline & BAF 18 & & BAF 2 & & & BAF 37 & & & \\
\hline $\mathbf{n}^{\circ}$ & $120 / 1$ & $122 / 1$ & $96 / 1$ & $102 / 1$ & $108 / 1$ & $59 / 1$ & $63 / 1$ & & \\
\hline Position & $\mu$ & $\mu$ & $\mu$ & $\mu$ & $\mu$ & $\phi$ & $\phi$ & $\phi$ & $\phi$ \\
\hline $\mathrm{SiO}_{2}$ & 47.98 & 47.18 & 50.20 & 49.53 & 50.37 & 50.91 & 49.56 & 51.38 & 50.07 \\
\hline $\mathrm{TiO}_{2}$ & 2.79 & 3.25 & 1.83 & 1.93 & 1.67 & 0.53 & 1.07 & 0.61 & 0.99 \\
\hline $\mathrm{Al}_{2} \mathrm{O}_{3}$ & 4.04 & 4.89 & 2.28 & 2.53 & 2.25 & 0.97 & 2.55 & 0.99 & 1.60 \\
\hline $\mathrm{Cr}_{2} \mathrm{O}_{3}$ & 0.00 & 0.00 & 0.00 & 0.00 & 0.00 & 0.00 & 0.00 & 0.00 & 0.00 \\
\hline $\mathrm{FeO}$ & 9.73 & 9.89 & 10.88 & 10.93 & 10.75 & 13.95 & 13.43 & 13.76 & 14.16 \\
\hline $\mathrm{MnO}$ & 0.23 & 0.24 & 0.34 & 0.37 & 0.33 & 0.50 & 0.34 & 0.50 & 0.49 \\
\hline $\mathrm{MgO}$ & 12.85 & 12.67 & 12.42 & 12.53 & 11.94 & 10.77 & 10.17 & 11.14 & 10.54 \\
\hline $\mathrm{CaO}$ & 21.39 & 21.17 & 21.72 & 21.20 & 21.96 & 21.81 & 21.84 & 21.56 & 21.47 \\
\hline $\mathrm{Na}_{2} \mathrm{O}$ & 0.56 & 0.71 & 0.64 & 0.60 & 0.69 & 0.58 & 0.94 & 0.53 & 0.72 \\
\hline $\mathrm{K}_{2} \mathrm{O}$ & 0.00 & 0.01 & 0.02 & 0.00 & 0.02 & 0.00 & 0.00 & 0.01 & 0.00 \\
\hline $\mathrm{P}_{2} \mathrm{O}_{5}$ & 0.10 & 0.18 & 0.01 & 0.00 & 0.02 & 0.00 & 0.00 & 0.00 & 0.04 \\
\hline $\mathrm{NiO}$ & 0.00 & 0.00 & 0.00 & 0.00 & 0.01 & 0.00 & 0.00 & 0.00 & 0.00 \\
\hline Total & 99.68 & 100.20 & 100.33 & 99.61 & 100.02 & 100.02 & 99.90 & 100.49 & 100.07 \\
\hline \multicolumn{10}{|c|}{ Formula based on 4 cations and 6 oxygen atoms } \\
\hline $\mathrm{Si}$ & 1.81 & 1.77 & 1.88 & 1.87 & 1.90 & 1.94 & 1.88 & 1.94 & 1.91 \\
\hline $\mathrm{Al}^{\mathrm{iv}}$ & 0.18 & 0.22 & 0.10 & 0.11 & 0.10 & 0.04 & 0.11 & 0.04 & 0.07 \\
\hline $\mathrm{Al}^{\mathrm{vi}}$ & 0.00 & 0.00 & 0.00 & 0.00 & 0.00 & 0.00 & 0.00 & 0.00 & 0.00 \\
\hline Alt & 0.18 & 0.22 & 0.10 & 0.11 & 0.10 & 0.04 & 0.11 & 0.04 & 0.07 \\
\hline $\mathrm{Ti}$ & 0.08 & 0.09 & 0.05 & 0.05 & 0.05 & 0.02 & 0.03 & 0.02 & 0.03 \\
\hline $\mathrm{Cr}$ & 0.00 & 0.00 & 0.00 & 0.00 & 0.00 & 0.00 & 0.00 & 0.00 & 0.00 \\
\hline $\mathrm{Fe}^{3+}$ & 0.09 & 0.12 & 0.08 & 0.08 & 0.06 & 0.09 & 0.13 & 0.07 & 0.11 \\
\hline $\mathrm{Fe}^{2+}$ & 0.21 & 0.19 & 0.26 & 0.26 & 0.27 & 0.35 & 0.30 & 0.36 & 0.34 \\
\hline $\mathrm{Mn}$ & 0.01 & 0.01 & 0.01 & 0.01 & 0.01 & 0.02 & 0.01 & 0.02 & 0.02 \\
\hline $\mathrm{Mg}$ & 0.72 & 0.71 & 0.69 & 0.71 & 0.67 & 0.61 & 0.58 & 0.63 & 0.60 \\
\hline $\mathrm{Ca}$ & 0.86 & 0.85 & 0.87 & 0.86 & 0.89 & 0.89 & 0.89 & 0.87 & 0.88 \\
\hline $\mathrm{Na}$ & 0.04 & 0.05 & 0.05 & 0.04 & 0.05 & 0.04 & 0.07 & 0.04 & 0.05 \\
\hline $\mathrm{K}$ & 0.00 & 0.00 & 0.00 & 0.00 & 0.00 & 0.00 & 0.00 & 0.00 & 0.00 \\
\hline Total & 4.00 & 4.00 & 4.00 & 4.00 & 4.00 & 4.00 & 4.00 & 4.00 & 4.00 \\
\hline $\mathrm{Mg} \#$ & 77.22 & 78.82 & 72.66 & 72.88 & 70.95 & 63.56 & 65.81 & 63.35 & 63.86 \\
\hline Wo & 49.24 & 49.74 & 49.37 & 51.94 & 50.22 & 46.78 & 45.02 & 44.39 & 43.64 \\
\hline En & 37.82 & 37.71 & 37.63 & 33.16 & 36.47 & 37.66 & 40.25 & 39.96 & 40.69 \\
\hline Fs & 12.93 & 12.54 & 13.00 & 14.90 & 13.31 & 15.56 & 14.73 & 15.65 & 15.67 \\
\hline
\end{tabular}


evolution (Figure 9): in one side the enrichment in $\mathrm{FeO}$ and $\mathrm{MgO}$ from rim to core (Figure 9(C) and Figure 9(E)) and in another side the enrichment of $\mathrm{TiO}_{2}$ and $\mathrm{CaO}$ from core to rim (Figure 9(B) and Figure 9(D)), with the decrease of $\mathrm{Al}_{2} \mathrm{O}_{3}$ and $\mathrm{Na}_{2} \mathrm{O}$ from core to rim (Figure $9(\mathrm{~A})$ and Figure $9(\mathrm{~F})$ ).

Clinopyroxene crystals core in basanite BAF 22 and in alkali basalt BAF 36 are more Mg-rich than the rim. Ti is higher in the rim compared to the core (Table 3). This observation is also made with the $\mathrm{Al}^{\mathrm{iv}}$. Notice that Mn contents are lower from rim to core. Zoning clinopyroxenes are rim $\mathrm{Fe}^{3+}$ rich than the core. $\mathrm{Al}^{\mathrm{vi}}$ and $\mathrm{Cr}$ are higher from core to rim. The cores in phenocrysts are Si rich (1.71 1.79 a.p.f.u.) compared to the rim (1.70 - 1.74 a.p.f.u.). In addition the $\mathrm{Al} / \mathrm{Ti}$ ratio is low at the rim $(2.78-3.49)$ and high in the core (3.63 - 13.10).

\subsubsection{Fe-Ti Oxides}

Structural formula of oxides has been calculated according the [32] method and the percentage of ulvospinel according method [33]. Results are listed in Table 4. In all the studied lavas oxides are represented by phenocrysts, microphenocrysts and microcrysts. All these oxides are magnetite and belong to the
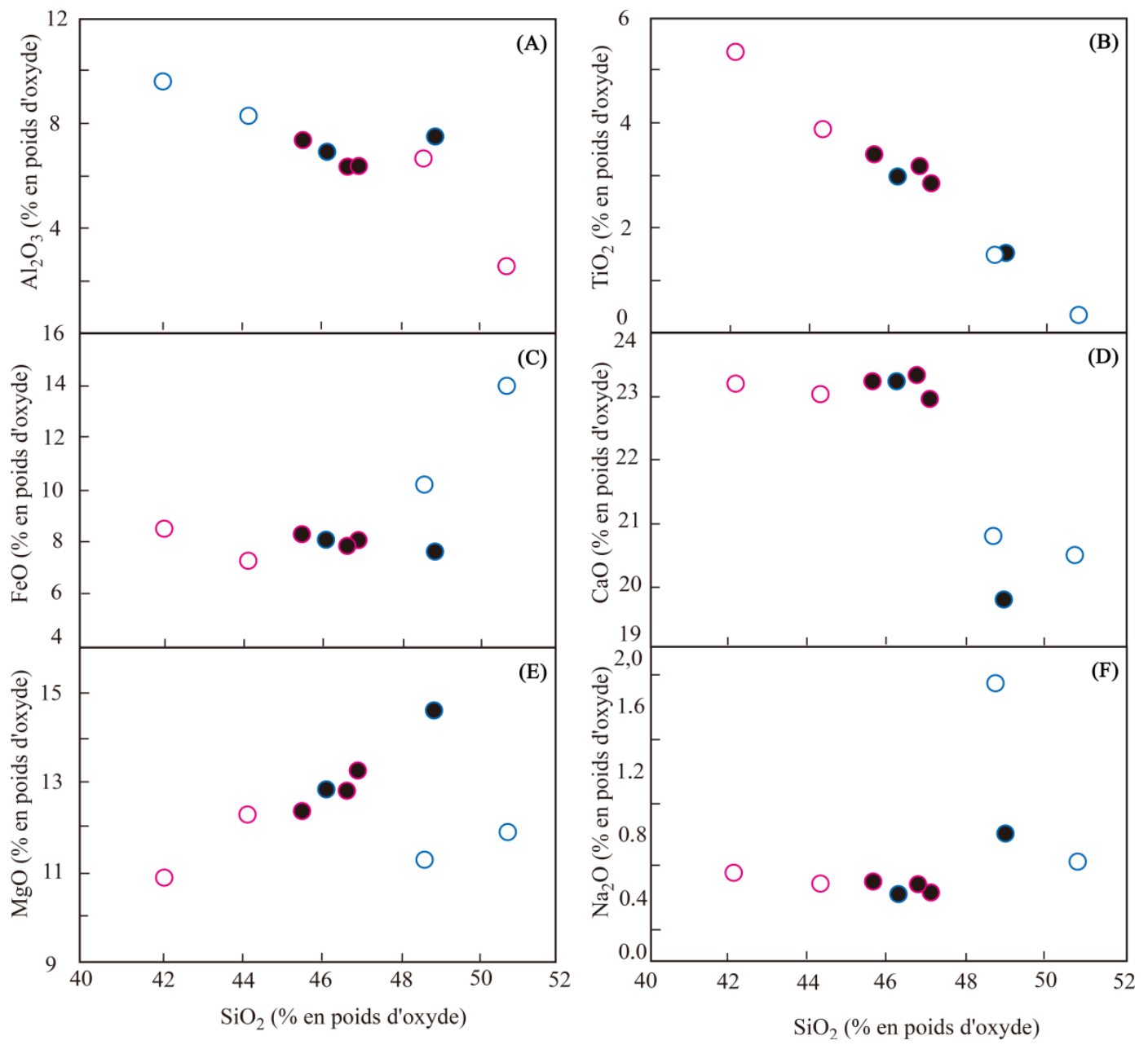

Figure 9. Relations between $\mathrm{Al}_{2} \mathrm{O}_{3}, \mathrm{TiO}_{2}, \mathrm{FeO}, \mathrm{Na}_{2} \mathrm{O}, \mathrm{MgO}$ and $\mathrm{SiO}_{2}$ of zoning $\mathrm{Cpx}$ in alkaline basaltic lavas of Bafang and its environs. Black circle (basanites). White circle (alkali basalt). Blue (core). Pink (rim). 
Table 4. Representative microprobe analyses of Fe-Ti oxide from alkaline lavas from the Bafang and its environs. USP = Uvöspinel; $\mathrm{Sp}=$ Spinel; $\mathrm{Mt}=$ Magnetite.

\begin{tabular}{|c|c|c|c|c|c|c|c|c|c|c|c|c|c|}
\hline \multirow{3}{*}{$\begin{array}{c}\text { Type of } \\
\text { rock } \\
\text { Name } \\
\text { Sample }\end{array}$} & \multicolumn{3}{|c|}{$\begin{array}{l}\text { High Mg Nb-enriched basalts } \\
\text { (LMg-NB) }\end{array}$} & \multicolumn{4}{|c|}{$\begin{array}{l}\text { Low Mg Nb-enriched basalts } \\
\text { (LMg-NB) }\end{array}$} & \multirow[b]{2}{*}{ Hawaiite } & \multirow{2}{*}{\multicolumn{2}{|c|}{ Mugearite }} & \multirow{3}{*}{\multicolumn{2}{|c|}{ BAF37 }} & \multirow[b]{4}{*}{$\mu$} \\
\hline & Basanite & & Basalt & Basalt & & & & & & & & & \\
\hline & BAF 22 & & BAF 42 & BAF 18 & BAF36 & & & BAF 2 & & BAF3 & & & \\
\hline Position & $\mu$ & $\mu$ & $\mu$ & $\mu$ & $\mu \phi$ & $\mu$ & $\mu$ & $\mu \phi$ & $\mu \phi$ & $\phi$ & $\mu$ & $\phi$ & \\
\hline $\mathrm{n}^{\circ}$ & $23 / 1$ & $29 / 1$ & $83 / 1$ & $123 / 1$ & $51 / 1$ & $42 / 1$ & $48 / 1$ & $103 / 1$ & $105 / 1$ & $114 / 1$ & $111 / 1$ & $71 / 1$ & $61 / 1$ \\
\hline $\mathrm{SiO}_{2}$ & 0.10 & 0.03 & 0.25 & 0.05 & 0.06 & 0.05 & 0.12 & 0.06 & 0.04 & 0.12 & 0.09 & 0.06 & 0.10 \\
\hline $\mathrm{Al}_{2} \mathrm{O}_{3}$ & 2.57 & 2.55 & 4.75 & 2.07 & 3.30 & 1.32 & 3.26 & 1.81 & 1.72 & 2.56 & 1.61 & 1.50 & 1.51 \\
\hline $\mathrm{TiO}_{2}$ & 25.14 & 24.86 & 22.90 & 21.89 & 22.79 & 25.99 & 22.20 & 26.17 & 26.15 & 21.79 & 23.05 & 20.38 & 19.93 \\
\hline $\mathrm{FeO}$ & 65.30 & 65.30 & 62.85 & 68.56 & 66.16 & 67.28 & 66.62 & 67.06 & 67.96 & 69.56 & 69.91 & 73.37 & 72.90 \\
\hline $\mathrm{MnO}$ & 0.72 & 0.76 & 0.58 & 0.60 & 0.59 & 0.71 & 0.56 & 0.71 & 0.80 & 0.70 & 0.71 & 0.72 & 0.79 \\
\hline $\mathrm{MgO}$ & 3.69 & 3.07 & 5.07 & 3.23 & 3.79 & 1.98 & 4.09 & 2.20 & 2.22 & 2.70 & 2.37 & 1.50 & 1.11 \\
\hline $\mathrm{CaO}$ & 0.19 & 0.14 & 0.22 & 0.13 & 0.02 & 0.16 & 0.05 & 0.00 & 0.00 & 0.00 & 0.00 & 0.01 & 0.05 \\
\hline $\mathrm{Cr}_{2} \mathrm{O}_{3}$ & 0.27 & 0.25 & 0.37 & 0.40 & 0.58 & 0.14 & 0.46 & 0.00 & 0.00 & 0.00 & 0.00 & 0.00 & 0.00 \\
\hline $\mathrm{NiO}$ & 0.03 & 0.09 & 0.04 & 0.00 & 0.02 & 0.00 & 0.00 & 0.00 & 0.00 & 0.00 & 0.00 & 0.00 & 0.00 \\
\hline $\mathrm{Na}_{2} \mathrm{O}$ & 0.02 & 0.02 & 0.00 & 0.00 & 0.07 & 0.01 & 0.00 & 0.00 & 0.00 & 0.00 & 0.06 & 0.05 & 0.00 \\
\hline $\mathrm{K}_{2} \mathrm{O}$ & 0.02 & 0.01 & 0.00 & 0.01 & 0.01 & 0.03 & 0.00 & 0.01 & 0.00 & 0.00 & 0.00 & 0.00 & 0.00 \\
\hline $\mathrm{P}_{2} \mathrm{O}_{5}$ & 0.00 & 0.00 & 0.00 & 0.03 & 0.02 & 0.02 & 0.05 & 0.00 & 0.00 & 0.00 & 0.00 & 0.00 & 0.00 \\
\hline TOTAL & 97.76 & 96.84 & 96.66 & 96.57 & 96.84 & 97.57 & 96.95 & 98.04 & 98.88 & 97.44 & 97.81 & 97.60 & 96.41 \\
\hline \multicolumn{14}{|c|}{ Formula based on 24 cations and 32 oxygen atoms } \\
\hline $\mathrm{Si}$ & 0.0288 & 0.0098 & 0.0702 & 0.0138 & 0.0173 & 0.0161 & 0.0334 & 0.0189 & 0.0115 & 0.0357 & 0.0266 & 0.0162 & 0.0296 \\
\hline $\mathrm{Al}$ & 0.8739 & 0.8790 & 1.5938 & 0.7150 & 1.1213 & 0.4604 & 1.1056 & 0.6259 & 0.5905 & 0.8803 & 0.5561 & 0.5207 & 0.5331 \\
\hline $\mathrm{Ti}$ & 5.4512 & 5.4690 & 4.9067 & 4.8125 & 4.9431 & 5.7678 & 4.8071 & 5.7701 & 5.7176 & 4.7773 & 5.0695 & 4.5140 & 4.4845 \\
\hline $\mathrm{Fe}^{3+}$ & 4.1182 & 4.1202 & 4.3707 & 5.5436 & 4.8691 & 3.9591 & 5.1086 & 3.8008 & 3.9513 & 5.4937 & 5.2875 & 6.4496 & 6.4400 \\
\hline $\mathrm{Cr}$ & 0.0626 & 0.0576 & 0.0829 & 0.0930 & 0.1333 & 0.0328 & 0.1048 & 0.0000 & 0.0000 & 0.0000 & 0.0000 & 0.0000 & 0.0000 \\
\hline $\mathrm{Fe}^{2+}$ & 11.6264 & 11.8562 & 10.6052 & 11.2217 & 11.0879 & 12.6452 & 10.9335 & 12.6415 & 12.5721 & 11.4657 & 11.8135 & 11.6236 & 11.7992 \\
\hline $\mathrm{Mg}$ & 1.5842 & 1.3397 & 2.1540 & 1.4060 & 1.6283 & 0.8715 & 1.7539 & 0.9612 & 0.9601 & 1.1737 & 1.0334 & 0.6605 & 0.4954 \\
\hline $\mathrm{Mn}$ & 0.1751 & 0.1892 & 0.1398 & 0.1481 & 0.1443 & 0.1776 & 0.1369 & 0.1769 & 0.1968 & 0.1737 & 0.1766 & 0.1808 & 0.2013 \\
\hline $\mathrm{Ni}$ & 0.0065 & 0.0220 & 0.0084 & 0.0000 & 0.0035 & 0.0000 & 0.0000 & 0.0000 & 0.0000 & 0.0000 & 0.0000 & 0.0000 & 0.0000 \\
\hline TOTAL & 24 & 24 & 24 & 24 & 24 & 24 & 24 & 24 & 24 & 24 & 24 & 24 & 24 \\
\hline USP & 90 & 88 & 88 & 83 & 85 & 89 & 85 & 89 & 89 & 81 & 83 & 75 & 75 \\
\hline $\mathrm{Sp}$ & 4 & 4 & 7 & 3 & 5 & 2 & 5 & 2 & 2 & 4 & 2 & 2 & 2 \\
\hline Mt & 6 & 8 & 5 & 13 & 11 & 9 & 10 & 8 & 9 & 15 & 14 & 22 & 23 \\
\hline
\end{tabular}

titanomagnetite serie forming the solid solution between magnetite $\left(\mathrm{Fe}_{3} \mathrm{O}_{4}\right)$ and ulvospinel $\left(\mathrm{Fe}_{2} \mathrm{TiO}_{4}\right)$ (Figure 10). These oxides are $\mathrm{FeO}$ high $(65.30 \mathrm{wt} \%-72.90$ wt\%) if regarding the $\mathrm{TiO}_{2}(19.93 \mathrm{wt} \%-26.17 \mathrm{wt} \%)$ contents. Their chemical 


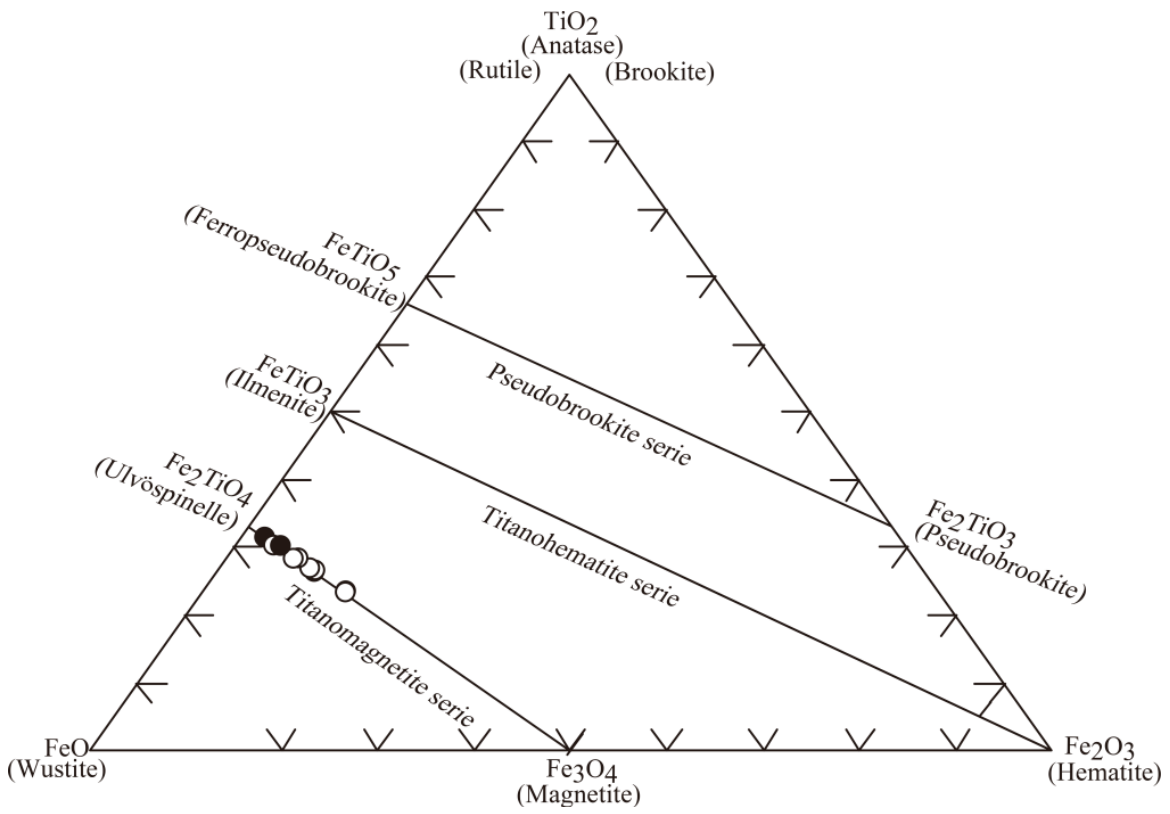

Figure 10. Position of oxide opaque of alkaline basaltic lavas from Bafang and its environs in $\mathrm{TiO}_{2}-\mathrm{FeO}-\mathrm{Fe}_{2} \mathrm{O}_{3}$ diagram of [34]. Symbols as in Figure 5.

compositions are close to ulvospinel $\left(\mathrm{Usp}_{74.84-90.19} \mathrm{Mt}_{5.23-22.94} \mathrm{Sp}_{1.77-7.12}\right)$ (Figure 10). The $\mathrm{TiO}_{2}$ (19.93 wt\% - $\left.26.15 \mathrm{wt} \%\right), \mathrm{MnO}(0.56 \mathrm{wt} \%-0.79$ wt.\%), $\mathrm{FeO}(62.85$ wt $\%$ - $73.37 \mathrm{wt} \%), \mathrm{Na}_{2} \mathrm{O}$ (0.00 wt\% - $\left.0.07 \mathrm{wt} \%\right), \mathrm{K}_{2} \mathrm{O}(0.00 \mathrm{wt} \%-0.02 \mathrm{wt} \%)$ and $\mathrm{P}_{2} \mathrm{O}_{5}(0.00 \mathrm{wt} \%-0.02 \mathrm{wt} \%)$ contents decrease whereas the $\mathrm{MgO}(1.11 \mathrm{wt} \%-5.07$ wt $\%), \mathrm{CaO}(0.00 \mathrm{wt} \%-0.22 \mathrm{wt} \%)$ et $\mathrm{Cr}_{2} \mathrm{O}_{3}(0.00 \mathrm{wt} \%-0.58 \mathrm{wt} \%)$ contents increase. The $\mathrm{Al}_{2} \mathrm{O}_{3}(1.32 \mathrm{wt} \%-4.75 \mathrm{wt} \%)$ contents are relatively constant.

\subsubsection{Feldspars}

Representative analyses of feldspars are listed in Table 5 and plotted in Figure 11. Feldspars in HMg-B are only plagioclase $\left(\mathrm{An}_{67.97-47.94} \mathrm{Ab}_{40.41-30.43} \mathrm{Or}_{11.65-1.60}\right)$. In $\mathrm{LMg}-\mathrm{B}$, feldspars are plagioclase $\left(\mathrm{An}_{64.75-15.84} \mathrm{Ab}_{69.19-34.63} \mathrm{Or}_{20.59-1.51}\right)$ and anorthoclase $\left(\mathrm{Ab}_{68.11-61.20} \mathrm{Or}_{33.87-20.91} \mathrm{An}_{10.98-4.93}\right)$. Plagioclases in $\mathrm{HMg}-\mathrm{B}$ are labradorite $\left(\mathrm{An}_{67.97-59.30} \mathrm{Ab}_{38.74-30.43} \mathrm{Or}_{2.57-1.60}\right)$ and andesine $\left(\mathrm{An}_{47.94} \mathrm{Ab}_{40.41} \mathrm{Or}_{11.65}\right)$. In LMg-B, plagioclases are labradorite $\left(\mathrm{An}_{64.75-51.96} \mathrm{Ab}_{44.98-33.72} \mathrm{Or}_{3.06-1.51}\right)$, andesine $\left(\mathrm{An}_{45.44-31.82}\right.$ $\left.\mathrm{Ab}_{62.66-51.79} \mathrm{Or}_{5.52-2.77}\right)$ and oligoclase $\left(\mathrm{An}_{26.65-15.84} \mathrm{Ab}_{69.19-63.57} \mathrm{Or}_{20.59-8.55}\right)$. Plagioclases are more abundant than anorthoclase. Only two microcrystals analyzed correspond to anorthoclase in mugearite.

The $\mathrm{CaO}$ content $(10.32 \mathrm{wt} \%-14.02 \mathrm{wt} \%$ and $1.06 \mathrm{wt} \%-13.46 \mathrm{wt} \%$ for $\mathrm{HMg}-\mathrm{B}$ and $\mathrm{LMg}-\mathrm{B}$ respectively) shows a wide range and the anorthite content ranges from $47.94 \mathrm{wt} \%$ to $67.97 \mathrm{wt} \%$ in $\mathrm{HMg}-\mathrm{B}$ and from $4.93 \mathrm{wt} \%$ to $64.75 \mathrm{wt} \%$ in $\mathrm{LMg}-\mathrm{B}$. The $\mathrm{Al}_{2} \mathrm{O}_{3}$ and $\mathrm{FeO}$ contents show wide compositional ranges with $28.88 \mathrm{wt} \%-31.11 \mathrm{wt} \%$; and $0.57-0.93 \mathrm{wt}$ in $\mathrm{HMg}-\mathrm{B}$. The values of $\mathrm{Al}_{2} \mathrm{O}_{3}(20.01$ wt $\%-30.27 \mathrm{wt} \%)$ and $\mathrm{FeO}(0.15 \mathrm{wt} \%-1.34 \mathrm{wt} \%)$ in $\mathrm{LMg}-\mathrm{B}$ in contrary show narrow compositional ranges.

The zoning observed on the plaglioclase in the alkali basalt BAF 36 indicates decreasing in $\mathrm{Al}$ and $\mathrm{Ca}$ and increasing in $\mathrm{Na}$ and $\mathrm{K}$ from rim to core (Figure 12). 


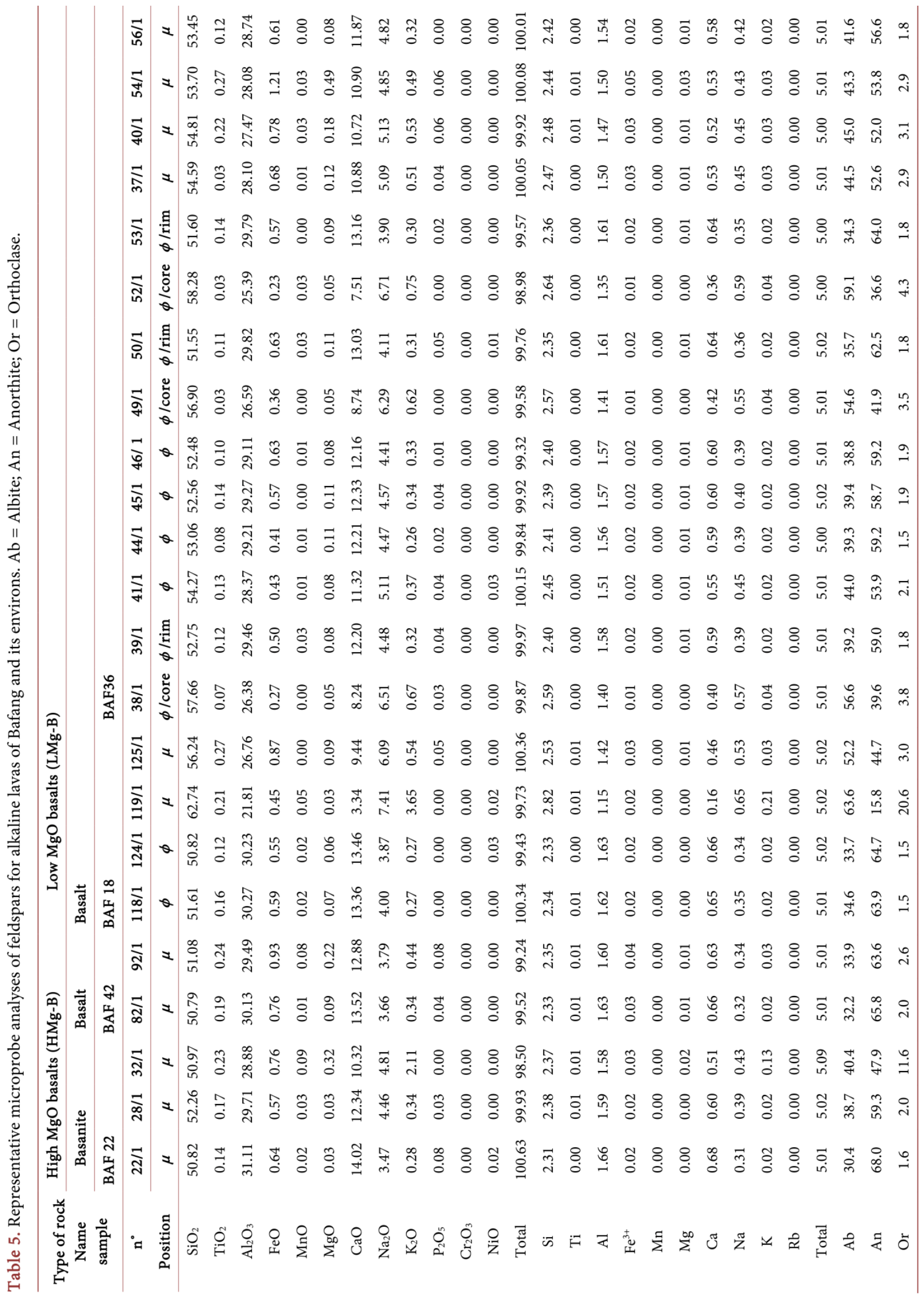




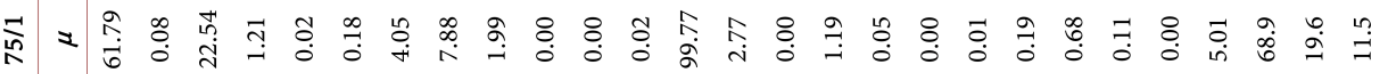

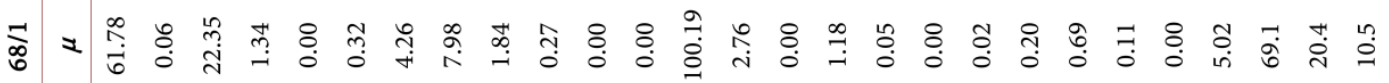

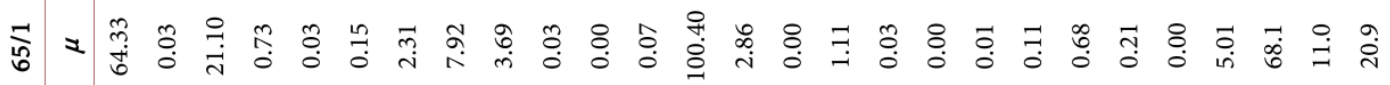

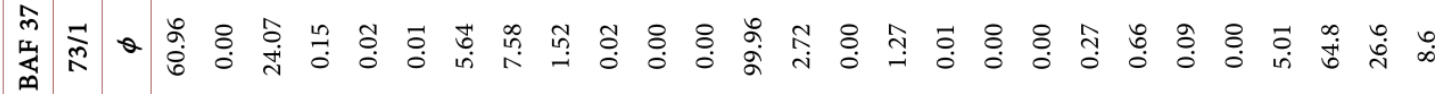
氛 $=$ 每

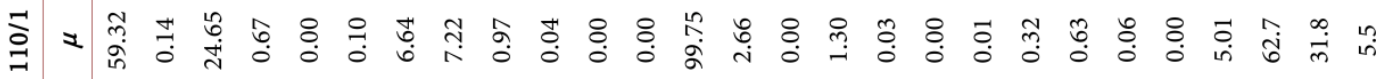
:

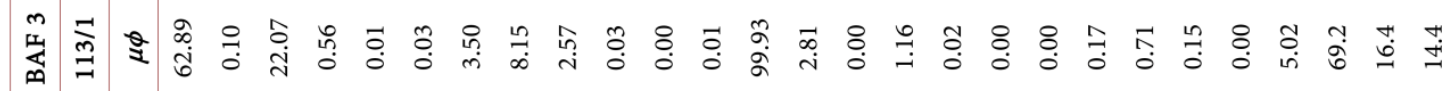

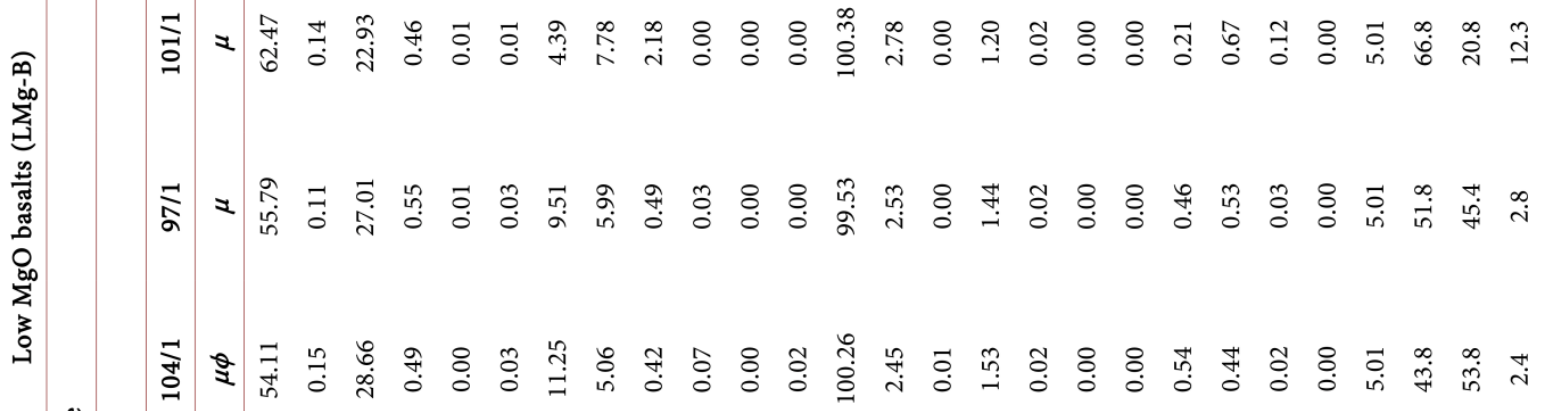
军

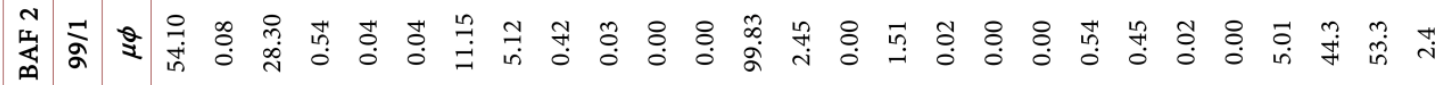
兽

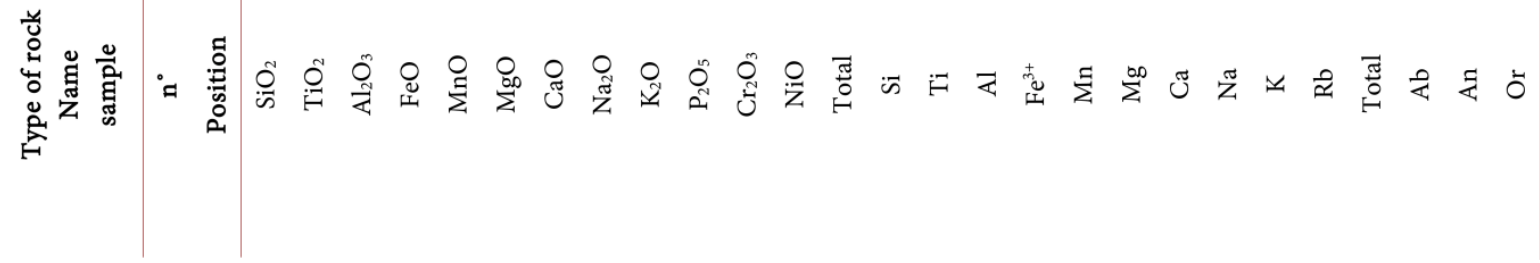




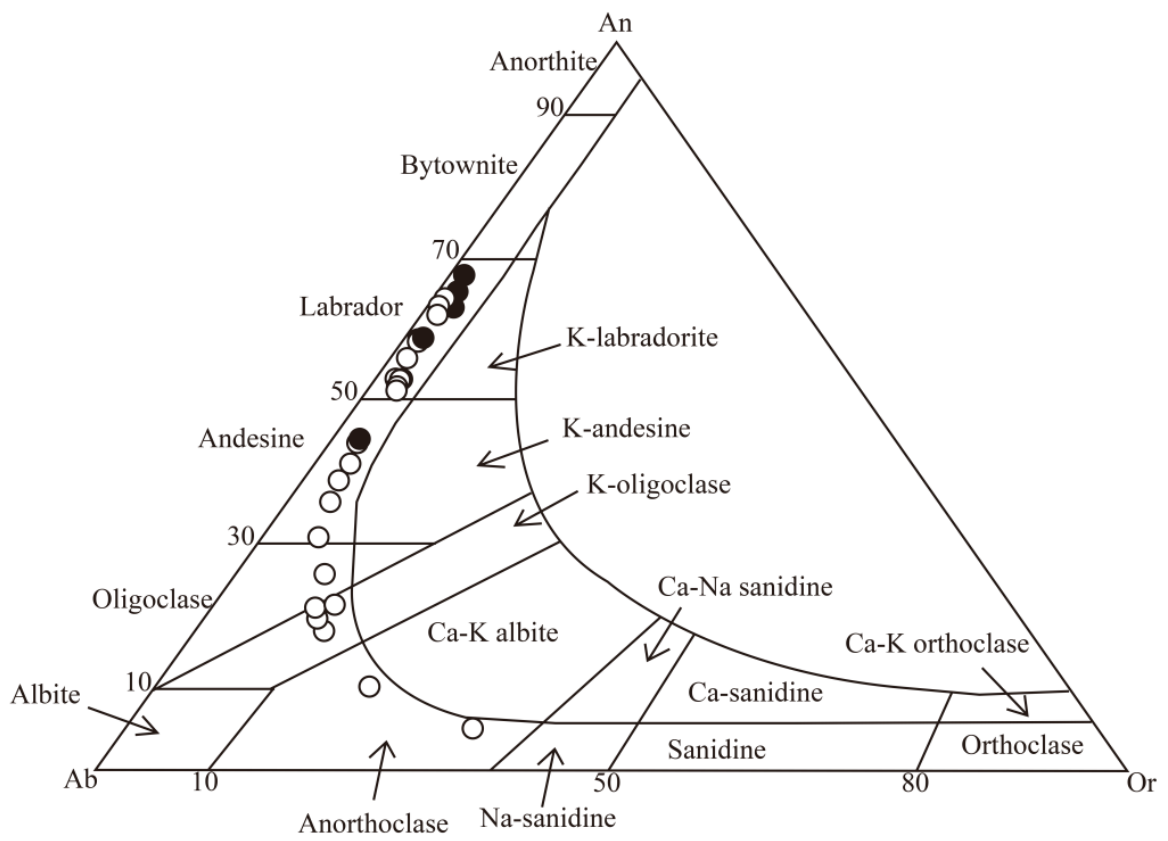

Figure 11. Position of feldspars of alkaline basaltic lavas Bafang in An-Ab-Or diagram of [35]. Symbols as in Figure 5.
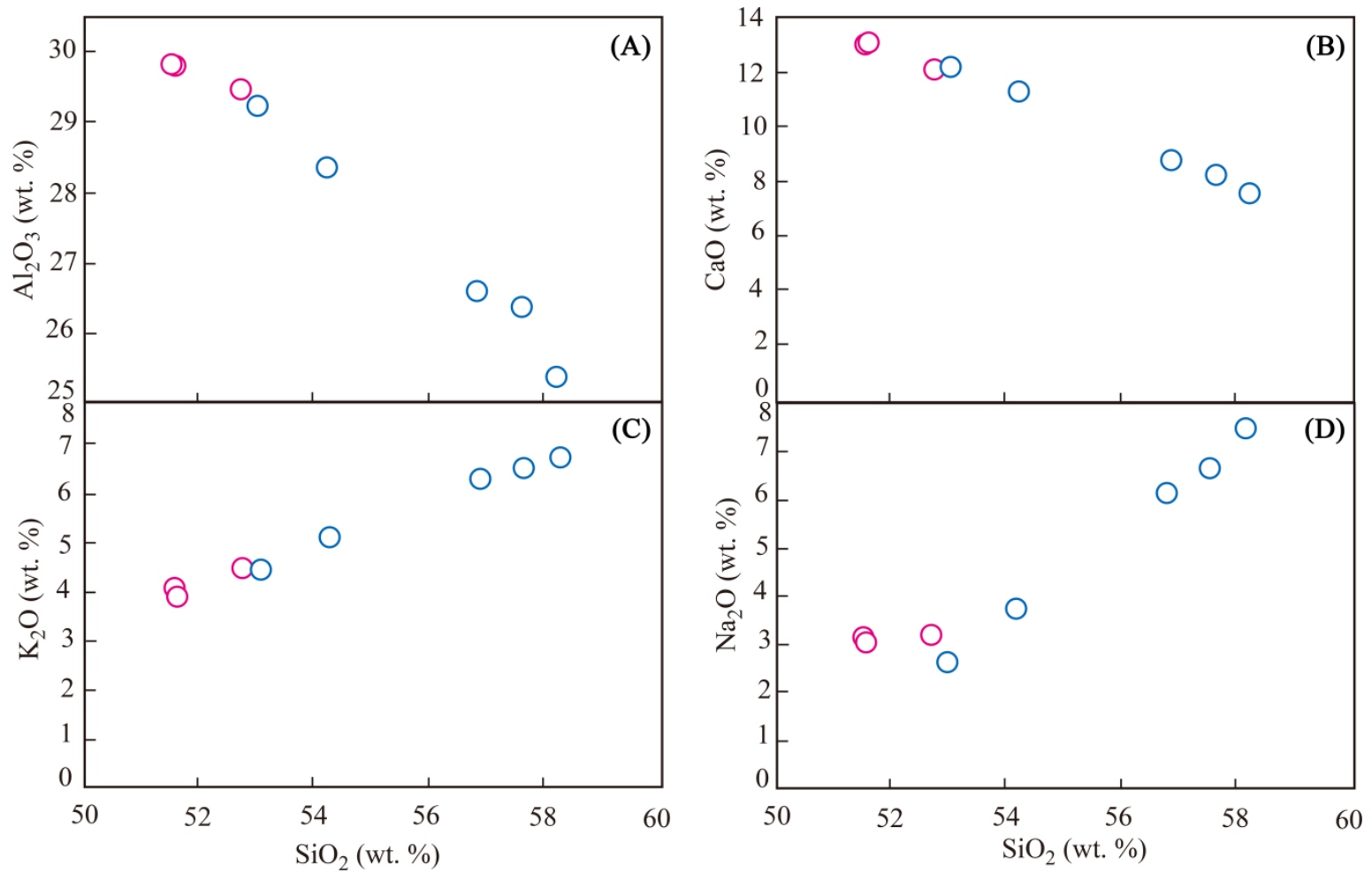

Figure 12. Relations between $\mathrm{Al}_{2} \mathrm{O}_{3}, \mathrm{CaO}, \mathrm{K}_{2} \mathrm{O}, \mathrm{Na}_{2} \mathrm{O}$, and $\mathrm{SiO}_{2}$ of zoning plagioclases in alkaline basaltic lavas of $\mathrm{Ba}-$ fang and its environs. Blue (core), Pink (rim).

This depletion causes at the end reverse zoning illustrated by the passage in some crystal from andesite $\left(\mathrm{Ab}_{55-59} \mathrm{An}_{37-42} \mathrm{Or}_{2-4}\right)$ composition in core to labrador $\left(\mathrm{An}_{54-59} \mathrm{Ab}_{39-44} \mathrm{Or}_{1-2}\right)$ composition in rim. 


\section{Discussion}

\subsection{Fractional Crystallization}

Many petrographical, mineralogical and geochemical features favor the fact that fractional crystallization is the main process responsable in the variation of compositions and the lithology diversity of basaltic lavas from area of Bafang. In the same order, the samples have highly variable compatible element contents. For example, Cr ranges from 1.6 to $515 \mathrm{ppm}$ and $\mathrm{Ni}$ from 1.1 to $261 \mathrm{ppm}$, and their content decreases with increasing $\mathrm{Mg} \#$ [2], indicating that fractionation of olivine and clinopyroxene are the main factors controlling the compatible element abundances. $\mathrm{Ni}, \mathrm{Cr}$ and Co contents in the most mafic lavas are generally smaller than the values assumed for primary magmas (Ni: 300 - 400 ppm; Cr: 300 - 500 ppm; Co: 50 - 70 ppm; e.g., [36] [37]), but are still high enough to indicate that for these samples the fractionation was limited.

Factional crytallazation process can be visible petrographically for the basaltic lavas from Bafang. This evolution by fractional crystallization [38] of studied rocks is corroborated by: the oxide inclusions observed in olivine, clinopyroxene and plagioclase (Figure 4); parallelism observed in the REE patterns [2], the variations in chemical compositions of phenocrysts fom core to rim (Figure 6, Figure 7, Figure 9, Figure 11; Tables 2-5) and the correlations observed in the Harker diagrammes [2] are also consistent with the fractional crystallization. The Mg\# values vary from 86 to 65 in the $\mathrm{HMg}-\mathrm{B}$ and from 53 to 38 in the $\mathrm{LMg}-\mathrm{B}$ [2]. These values indicate that olivine in the $\mathrm{HMg}$ - $\mathrm{B}$ are more magnesian $\left(\mathrm{Fo}_{87-65}\right.$, with $\mathrm{Fo}=\left[\mathrm{Mg} /\left(\mathrm{Mg}+\mathrm{Fe}^{2+}\right)\right.$. In contrary in the $\mathrm{LMg}-\mathrm{B}$, they are more ferrouferous $\left(\mathrm{Fo}_{53-38}\right)$. The variations observed with $\mathrm{Mg}$ \# from $\mathrm{HMg}-\mathrm{B}$ to $\mathrm{HMg}-\mathrm{B}$ also suggest fractional crystallization. In other hand, clinopyroxene crystals in studied lavas display the decreasing of $\mathrm{Ti}+\mathrm{Al}^{\mathrm{iv}}$ from basanites to mugearites suggesting that fractional crystallization occur at variable pressures ([39] [40]). Their Mg\# range from 63.35 to 89.52 and their $\mathrm{TiO}_{2}$ and $\mathrm{Al}_{2} \mathrm{O}_{3}$ contents vary between $0.31 \mathrm{wt} \%$ and $5.39 \mathrm{wt} \%$ and $0.97 \mathrm{wt} \%$ and $9.57 \mathrm{wt} \%$, respectively. $\mathrm{Ti}, \mathrm{Al}$ and both elements increase with iron enrichment (Table 3). Their increasing Ti with decreasing Mg\# reflects the normal fractionation trend (e.g. [41]). The $\mathrm{Cr}_{2} \mathrm{O}_{3}$ contents can reach $0.5 \mathrm{wt} \%$ but it sharply decreases with decreasing $\mathrm{Mg \#}$ (Table 3). Their $\mathrm{Ti} / \mathrm{Al}$ ratios $(0.08-0.51)$ are higher, while the $\mathrm{Al}^{\mathrm{vi}} / \mathrm{Al}^{\mathrm{lv}}$ ratios $(0.00-0.53)$ are lower. These ratios imply that they could have crystallized under various-pressure conditions (e.g. [42] [43]). Based on their slightly increasing $\mathrm{Ti} / \mathrm{Al}$ ratios during crystallization, they could have precipitated under continuously decreasing pressure. They could have been characterized by a significantly higher crystallization rate compared with the olivine phenocrysts (as suggested by Figure 9).

The ages obtained on the alkaline basaltic lavas of Bafang (10.46 - 6.27 Ma, [2] suggest that the geochemical diversity observed is explained by two distinctives magmatic series: basanite to basanite and hawaiite to mugearite.

The process of fractional crystallization has been modeling by using mass 
balance of sums square of major elements ([2] according to [44], after the calculation sheet of petro-mode. The model series have been calculated according to the process described by [45]. The trend showing the evolution of studied $\mathrm{HMg}-\mathrm{B}$ were tested as well as the trend of LMg-B. The results are reported in Tables 6-8. The first stage of crystallisation is that of basanite among them (BAF 22 à BAF 44), after a removal of $52.61 \%$ clinopyroxene $+20.55 \%$ plagioclase

Table 6. Least-square mass balance calculations (major elements) for derivation by factional crystallization of basanite from the basanite parent (BAF 22) to the basanite daughter (BAF 44). $\Sigma \mathrm{r}^{2}=$ sum of residual squares.

\begin{tabular}{|c|c|c|c|c|c|c|c|c|}
\hline Stage 1 & Parent BAF 22 & Daughter BAF 44 & Plagioclase & Clinopyroxene & Olivine & Magnetite & Calc. Parent & Residuals \\
\hline $\mathrm{SiO}_{2}$ & 43.03 & 43.91 & 50.82 & 38.06 & 38.06 & 0.10 & 42.99 & -0.04 \\
\hline $\mathrm{TiO}_{2}$ & 3.38 & 3.28 & 0.14 & 0.09 & 0.09 & 25.14 & 3.28 & -0.10 \\
\hline $\mathrm{Al}_{2} \mathrm{O}_{3}$ & 13.19 & 13.31 & 31.11 & 6.88 & 0.05 & 2.57 & 12.97 & -0.22 \\
\hline $\mathrm{Fe}_{2} \mathrm{O}_{3}$ & 1.84 & 1.81 & & 1.90 & & & 1.72 & -0.12 \\
\hline $\mathrm{FeO}$ & 12.20 & 12.09 & 0.64 & 5.26 & 24.64 & 65.30 & 12.38 & 0.18 \\
\hline $\mathrm{MnO}$ & 0.24 & 0.20 & 0.02 & 0.00 & 0.44 & 0.72 & 0.20 & -0.04 \\
\hline $\mathrm{MgO}$ & 10.61 & 10.19 & 0.03 & 12.25 & 37.32 & 3.69 & 10.43 & -0.18 \\
\hline $\mathrm{CaO}$ & 11.17 & 10.65 & 14.02 & 22.91 & 0.47 & 0.19 & 11.15 & -0.02 \\
\hline $\mathrm{Na}_{2} \mathrm{O}$ & 2.23 & 2.73 & 3.47 & 0.52 & 0.00 & 0.02 & 2.53 & 0.30 \\
\hline $\mathrm{K}_{2} \mathrm{O}$ & 1.49 & 1.29 & 0.28 & 0.00 & 0.01 & 0.02 & 1.15 & -0.34 \\
\hline $\mathrm{P}_{2} \mathrm{O}_{5}$ & 0.76 & 0.71 & 0.08 & 0.00 & 0.05 & 0.00 & 0.63 & -0.13 \\
\hline \multirow[t]{2}{*}{ Total } & 100.14 & 100.17 & 100.60 & 87.87 & 101.13 & 97.75 & 99.42 & \\
\hline & & & & & & & & $\Sigma r^{2}=0.37$ \\
\hline
\end{tabular}

Table 7. Least-square mass balance calculations (major elements) for derivation by factional crystallization of hawaiite to mugearite from the hawaiite parent (BAF 2) to the mugearite daughter (BAF 3). $\sum r^{2}=$ sum of residual squares.

\begin{tabular}{|c|c|c|c|c|c|c|c|c|}
\hline Stage 2 & Parent BAF 2 & Fils BAF 3 & Plagioclase & Clinopyroxene & Olivine & Magnetite & Calc. Parent & Residuals \\
\hline $\mathrm{SiO}_{2}$ & 49.58 & 50.36 & 55.79 & 50.20 & 34.33 & 0.06 & 49.21 & -0.37 \\
\hline $\mathrm{TiO}_{2}$ & 2.51 & 2.15 & 0.11 & 1.83 & 0.29 & 26.17 & 2.37 & -0.14 \\
\hline $\mathrm{Al}_{2} \mathrm{O}_{3}$ & 16.84 & 16.65 & 27.01 & 2.28 & 0.03 & 1.81 & 16.57 & -0.27 \\
\hline $\mathrm{Fe}_{2} \mathrm{O}_{3}$ & 1.75 & 1.80 & & & & & 1.56 & -0.19 \\
\hline $\mathrm{FeO}$ & 11.69 & 12.03 & 0.55 & 10.88 & 41.74 & 67.06 & 11.90 & 0.21 \\
\hline $\mathrm{MnO}$ & 0.23 & 0.24 & 0.01 & 0.34 & 1.00 & 0.71 & 0.23 & 0.00 \\
\hline $\mathrm{MgO}$ & 3.67 & 3.34 & 0.03 & 12.42 & 22.33 & 2.20 & 3.27 & -0.40 \\
\hline $\mathrm{CaO}$ & 5.99 & 5.44 & 9.51 & 21.72 & 0.45 & 0.00 & 6.02 & 0.03 \\
\hline $\mathrm{Na}_{2} \mathrm{O}$ & 4.82 & 4.73 & 5.99 & 0.64 & 0.03 & 0.00 & 4.57 & -0.25 \\
\hline $\mathrm{K}_{2} \mathrm{O}$ & 2.08 & 2.19 & 0.49 & 0.02 & 0.00 & 0.01 & 1.93 & -0.15 \\
\hline $\mathrm{P}_{2} \mathrm{O}_{5}$ & 1.02 & 1.24 & 0.03 & 0.01 & 0.16 & 0.00 & 1.08 & 0.06 \\
\hline \multirow[t]{2}{*}{ Total } & 100.18 & 100.17 & 99.52 & 100.34 & 100.36 & 98.02 & 98.69 & \\
\hline & & & & & & & & $\sum r^{2}=0.56$ \\
\hline
\end{tabular}


Table 8. Least-square mass balance calculations (major elements) for derivation by factional crystallization of hawaiite to mugearite from the hawaiite parent (BAF 2) to the mugearite daughter (BAF 41). $\Sigma r^{2}=$ sum of residual squares.

\begin{tabular}{ccccccccc}
\hline Stage 3 & Parent BAF 2 & Fils BAF 41 & Plagioclase & Clinopyroxene & Olivine & Magnetite & Calc. Parent & Residuals \\
\hline $\mathrm{SiO}_{2}$ & 49.58 & 50.51 & 55.79 & 50.20 & 34.33 & 0.06 & 49.95 & 0.37 \\
$\mathrm{TiO}_{2}$ & 2.51 & 2.20 & 0.11 & 1.83 & 0.29 & 26.17 & 2.48 & -0.03 \\
$\mathrm{Al}_{2} \mathrm{O}_{3}$ & 16.84 & 16.78 & 27.01 & 2.28 & 0.03 & 1.81 & 16.85 & 0.01 \\
$\mathrm{Fe}_{2} \mathrm{O}_{3}$ & 1.75 & 1.77 & & & & & 1.60 & -0.15 \\
$\mathrm{FeO}$ & 11.69 & 11.80 & 0.55 & 10.88 & 41.74 & 67.06 & 12.06 & 0.37 \\
$\mathrm{MnO}$ & 0.23 & 0.22 & 0.01 & 0.34 & 1.00 & 0.71 & 0.22 & -0.01 \\
$\mathrm{MgO}$ & 3.67 & 3.53 & 0.03 & 12.42 & 22.33 & 2.20 & 3.47 & -0.20 \\
$\mathrm{CaO}$ & 5.99 & 5.54 & 9.51 & 21.72 & 0.45 & 0.00 & 6.00 & 0.01 \\
$\mathrm{Na}_{2} \mathrm{O}$ & 4.82 & 4.50 & 5.99 & 0.64 & 0.03 & 0.00 & 4.44 & -0.38 \\
$\mathrm{~K}_{2} \mathrm{O}$ & 2.08 & 2.12 & 0.49 & 0.02 & 0.00 & 0.01 & 1.94 & -0.14 \\
$\mathrm{P}_{2} \mathrm{O}_{5}$ & 1.02 & 1.20 & 0.03 & 0.01 & 0.16 & 0.00 & 1.09 & 0.07 \\
$\mathrm{Total}^{2}$ & 100.18 & 100.17 & 99.52 & 100.34 & 100.36 & 98.02 & 100.10 & \\
& & & & & & & $5 \mathrm{r}^{2}=0.51$ \\
\hline
\end{tabular}

$+14.31 \%$ olivine $+12.54 \%$ de magnetite, thus the average of mineral fractionation of $11.5 \%$. The second stage is that of crystallization from hawaiite to mugearite (BAF 2 to BAF 3 and BAF 2 to BAF 41) after the removal of $(20.30 \%$ $22.43 \%)$ clinopyroxene $+(61.85 \%-63.35 \%)$ plagioclase $+(14.14 \%-17.85 \%)$ magnetite, thus the average of mineral fractionation of $13.5 \%$ and $9.7 \%$.

\subsection{Magma Storage and Recharge}

Before erupting at the surface, magma is often (although not always) stored in subsurface reservoirs, where chemical differntiation produces a variety of compositional products. The differences in bulk composition among analyzed basaltic lavas from Bafang and its environ [2], could be due to the short residence in the magma chambers. In fact, basaltic lavas display a large among of lava types from more basaltic (basanite) to less basaltic (mugearite). The distinction among these lavas could indicate different magma chambers from where they derived. Similarities can also be seen in mineral chemistry (Table 2, Table 3 and Table 5; Figure 6, Figure 7, Figure 9 and Figure 12) and optical appearance in thin sections with olivines, clinopyroxenes and plagioclases (Figure $4\left(\mathrm{~A}^{\prime}\right)$ and Figure $4\left(C^{\prime}\right)$ ) showing zonations. Taking into account these features mentioned above and combining them with temperature and pressure results from studied basaltic lavas, it is here seem plausible to suggest that there are magma storage which favor fractional crystallization.

During these periods of storage, interaction between injected primary melts and the shallow level reservoirs, could be suggested to cause chemical differentiation observed in olivine, clinopyroxene and plagioclase phenocrysts (Table 2, Table 3 and Table 5; Figure 6, Figure 7, Figure 9 and Figure 12) and probably 
their textural feature such as zonation (Figure $4\left(\mathrm{~A}^{\prime}\right)$ and Figure $4\left(\mathrm{C}^{\prime}\right)$ ). Zoned phenocrystals preserve fingerprints of magmatic processes over their life time ([46] [47]). Perturbations in composition, temperature and pressure can cause crystal zonation [48]. The textural feature and chemical composition of zoned crystals in the basaltic lavas from Bafang and its environs can be used to discriminate between different magmatic processes responsible for the zonation. Zoned crystals were subdivided into two categories: (i) concentric reverse-zoned (with $\mathrm{Mg}$ and $\mathrm{Ni}$-rich cores and $\mathrm{Fe}, \mathrm{Mn}$ and $\mathrm{Ca}$-rich rims in olivines and clinopyroxene; as well as $\mathrm{Al}$ and $\mathrm{Ca}$ in plagioclase; Table 2, Table 3 and Table 5); (ii) concentric normal zoned ( $\mathrm{Fe}, \mathrm{Mn}$ and Ca-rich cores and $\mathrm{Mg}$ and Ni-rich rims; Table 2 and Table 3). There is a relation between the style of zoning and the nature of the magmatic perturbation involving different magmas [48]. Normal zoning observed in studied lavas may be a consequence of rapid crystallization whereas reverse zoning can be produced as crystals resident in the chamber are subjected to heating by the intruding magma induced by recharge process [48].

\section{Conclusion}

Alkaline basaltic lavas of Bafang with Ne-Ol-Di-Hy normative are derived from low degree of partial melting of garnet peridotite and pyroxenite of lithospheric mantle. These rocks are mesocrate, melanocrate and holomelanocrate exhibiting phorphyritic, aphyritic and fluidal textures. They are made of olivine, pyroxene, oxides and feldspar. Olivines are magnesian and ferrouferous and are represented by chrysolite, hyalosierite and hortonolite. Pyroxenes are Ca-Mg-Fe clinopyroxenes represented by diopside and augite. Oxides are magnetite represented by ulvospinel in the titanomagnetite serie. Feldspars are plagioclase and anorthoclase. Plagioclases are calcic and sodic represented by labrador, andesine and oligoclase. The differentiation from basalts and basanites to mugearites is accounted by the removal of olivine, Clinopyroxene and oxides. The chemical variation from core to rim or rim to core of phenocrysts analyzed from the studied lavas in this work may indicate a less evolved primary magma and that of shallower chamber after the recharge.

\section{Acknowledgements}

We thank IRD (Institut de Recherche pour le Développement) for logistic support during field work in Cameroon.

\section{Conflicts of Interest}

The authors declare no conflicts of interest regarding the publication of this paper.

\section{References}

[1] Déruelle, B., Ngounouno, I. and Demaiffe, D. (2007) The "Cameroon Hot Line" (CHL): A Unique Example of Active Alkaline Intraplate Structure in Both Oceanic 
and Continental Lithospheres. Comptes Rendus Geoscience, 339, 589-600. https://doi.org/10.1016/j.crte.2007.07.007

[2] Tchuimegnie Ngongang, N.B., Kamgang, P., Chazot, G., Agranier, A., Bellon, H. and Nonnotte, P. (2015) Age, Geochemical Characteristics and Petrogenesis of Cenozoic Intraplate Alkaline Volcanic Rocks in the Bafang Region, West Cameroon. Journal of African Earth Sciences, 102, 218-232. https://doi.org/10.1016/j.jafrearsci.2014.10.011

[3] Njonfang, E., Nono, A., Kamgang, P., Ngako, V. and Tchoua, F.M. (2011) Cameroon Line Alkaline Magmatism (Central Africa): A Reappraisal. Geological Society of America Special Paper 478, 173-191. https://doi.org/10.1130/2011.2478(09)

[4] Burke, K. (2001) Origin of the Cameroon Line of Volcano-Capped Swells. Journal of Geology, 109, 349-362. https://doi.org/10.1086/319977

[5] Kampunzu, A. and Popoff, M. (1991) Distribution of Themain Phanerozoic African Rifts and Associated Magmatism, Introductory Notes. In: Kampunzu, A.B. and Lubala, R., Eds., Magmatism in Extensional Structural Settings. The Phanerozoic African Plate, Springer, Berlin, 2-10. https://doi.org/10.1007/978-3-642-73966-8_1

[6] Kagou Dongmo, A., Nkouathio, D., Nono, A., Kwekam, M., Tematio, P., Wandji, P., Tchoua, F., Pouclet, A. and Bourdier. J.-L. (2006) Discovery of Strombolian Cone at Totap-Tapsetsa, North-East Slope of Mount Bambouto: Implications in the Petrogenesis and the Volcanic Activity of Bambouto Volcano (Cameroon Line) Abstract. Cities on Volcanoes, 4, 23-27.

[7] Kamgang, P., Njonfang, E., Chazot, G. and Tchoua, F. (2007) Géochimie et géochronologie des laves felsiques des Monts Bamenda (Ligne Volcanique du Cameroun). Comptes Rendus Géoscience, 339, 659-666.

https://doi.org/10.1016/j.crte.2007.07.011

[8] Kamgang, P., Chazot, G., Njonfang, E. and Tchoua, F. (2008) Geochemistry and Geochronology of Mafic Rocks from Bamenda Mountains (Cameroon): Source Composition and Crustal Contamination along the Cameroon Volcanic Line. Comptes Rendus Geoscience, 340, 850-857. https://doi.org/10.1016/j.crte.2008.08.008

[9] Kamgang, P., Njonfang, E., Nono, A., Gountie Dedzo, M. and Tchoua, F.M. (2010) Petrogenesis of a Silicic Magma System: Geochemical Evidence from Bamenda Mountains, NW Cameroon, Cameroon Volcanic Line. Journal of African Earth Sciences, 58, 285-304. https://doi.org/10.1016/j.jafrearsci.2010.03.008

[10] Kamgang, P., Chazot, G., Njonfang, E., Tchuimegnie Ngongang, N.B. and Tchoua, F. (2013) Mantle Sources and Magma Evolution beneath the Cameroon Volcanic Line: Geochemistry of Mafic Rocks from the Bamenda Mountains (NW Cameroon). Gondwana Research, 24, 727-741. https://doi.org/10.1016/j.gr.2012.11.009

[11] Njilah, I.K. (1991) Geochemistry and Petrogenesis of Tertiary-Quaternary Volcanic Rocks from Oku-Ndu Area, N.W. Cameroon. Ph.D. Thesis, University of Leeds, Leeds, $350 \mathrm{p}$.

[12] Marzoli, A., Renne, P.R., Peccirillo, E.M., Castorina, F., Bellieni, G., Melfi, A.G., Nyobe, J.B. and N'ni, J. (1999) Silicic Magmas from the Continental Cameroon Volcanic Line (Oku, Bambouto and Ngaoundere): ${ }^{40} \mathrm{Ar}-{ }^{39} \mathrm{Ar}$ Dates, Petrology, Sr-Nd-O Isotopes and Their Petrogenetic Significance. Contributions to Mineralogy and Petrology, 135, 133-150. https://doi.org/10.1007/s004100050502

[13] Kagou Dongmo, A., Wandji, P., Pouclet, A., Vicat, J.P., Cheilletz, A., Nkouathio, D.G., Alexandrov, P. and Tchoua, F.M. (2001) Evolution volcanologique du mont Manengouba (Ligne du Cameroun), nouvelles données pétrographiques, géochimiques 
et géochronologiques. Comptes Rendus Académie des Sciences Paris, 333, 155-162. https://doi.org/10.1016/S1251-8050(01)01625-1

[14] Nana, J. (1988) Le complexe volcano-plutonique de Bana (OuestCameroun): géologie et pétrologie. Thèse de Doctorat $3^{\text {ème }}$ Cycle Université de Paris-Sud Orsay, 131 p.

[15] Kuepouo, G. (2004) Geology, Petrology and Geochemistry of the Tertiary Bana Volcano Plutonic Complex, Cameroon Line, Central Africa. Ph.D. Thesis, Kobe University, Kobe, 301 p.

[16] Kuepouo, G., Tchouankoue, J.P., Nagao, T. and Sato, H. (2006) Transitional Tholeiitic Basalts in the Tertiary Bana Volcano-Plutonic Complex, Cameroon Line. Journal of African Earth Sciences, 45, 318-332. https://doi.org/10.1016/j.jafrearsci.2006.03.005

[17] Fosso, J., Menard, J.-J., Bardintzeff, J.-M., Wandji, P., Tchoua, F.M. and Bellon, H. (2005) Les laves du mont Bangou: Une première manifestation volcanique éocène, à affinité transitionnelle, de la Ligne du Cameroun. Comptes Rendus Géoscience, 337, 315-325. https://doi.org/10.1016/j.crte.2004.10.014

[18] Djouka-Fonkwé, M.L., Schulz, B., Schüssler, U., Tchouankoué, J.-P. and Nzolang, C. (2008) Geochemistry of the Bafoussam Pan-African I- and S-Type Granitoids in Western Cameroon. Journal of African Earth Sciences, 50, 148-167. https://doi.org/10.1016/j.jafrearsci.2007.09.015

[19] Tchuimegnie Ngongang, N.B. (2016) Pétrologie, géochimie et géochronologie des laves basaltiques de Bafang et ses environs. Doctorat/PhD, Université de Yaoundé I, Yaoundé, 191p + annexes.

[20] Dumort, J.C. (1968) Cartes géologiques de reconnaissance à l'échelle de 1/50000. République Fédérale du Cameroun, Douala-Ouest. Bulletin de Recherches Géologiques et Minières, Direction des mines et de la géologie du Cameroun, et une notice explicative. $68 \mathrm{p}$.

[21] Cotten, J., Le Dez, A., Bau, M., Caroff, M., Maury, R.C., Dulski, P., Fourcade, S., Bohn, M. and Brousse, R. (1995) Origin of Anomalous Rare-Earth Element and Yttrium Enrichments in Subaerially Exposed Basalts: Evidence from French Polynesia. Chemical Geology, 119, 115-138. https://doi.org/10.1016/0009-2541(94)00102-E

[22] Li, Z.-X.A. and Lee, C.-T.A. (2006) Geochemical Investigation of Serpentinized Oceanic Lithospheric Mantle in the Feather River Ophiolite, California: Implications for the Recycling Rate of Water by Subduction. Chemical Geology, 235, 161-185. https://doi.org/10.1016/j.chemgeo.2006.06.011

[23] Defant, M.J., Richerson, M., de Boer, J.Z., Stewart, R.H., Maury, R.C, Bellon, H., Drummond, M.S., Feigenson, M.D. and Jackson, T.E. (1991) Dacite Genesis via Both Slab Melting and Differentiation: Petrogenesis of La Yeguada Volcanic Complex, Panama. Journal of Petrology, 32, 1101-1142.

https://doi.org/10.1093/petrology/32.6.1101

[24] Tamen, J., Nkoumbou, C., Mouafo, L., Reusser, E. and Tchoua, M.F. (2007) Petrology and Geochemistry of Monogenic Volcanoes of Barombi Koto Volcanic Field (Kumba Graben, Cameroon Volcanic Line): Implications for Mantle Source Characteristics. Comptes Rendus Geoscience, 339, 799-809.

https://doi.org/10.1016/j.crte.2007.09.007

[25] Nkouandou, F.O., Ngounouno, I., Déruelle, B., Ohnenstetter, D., Montigny, R. and Demaiffe, D. (2008) Petrology of the Mio-Pliocene Volcanism to North and East of Ngaoundéré (Adamawa, Cameroon). Comptes Rendus Geoscience, 340, 28-37. https://doi.org/10.1016/j.crte.2007.10.012

[26] Njonfang, E., Tchuente Tchoneng, G., Cozzupoli, D. and Lucci, F. (2013) Petroge- 
nesis of the Sabongari Alkaline Complex, Cameroon Line (Central Africa): Preliminary Petrological and Geochemical Constraints. Journal of African Earth Sciences, 83, 25-54. https://doi.org/10.1016/j.jafrearsci.2013.03.004

[27] Roeder, P.L. and Emslie, R.F. (1970) Olivine-Liquid Equilibrium. Contributions to Mineralogy and Petrology, 29, 275-289. https://doi.org/10.1007/BF00371276

[28] Hughes, C.J. (1982) Igneous Petrology. Elsevier, Amsterdam.

[29] Morimoto, N., Fabries, J., Ferguson, A.K., Ginzburg, I.V., Ross, M., Seifert, F.A., Zussmann, J., Aoki, K. and Gottardi, G. (1988) Nomenclature of Pyroxenes. American Mineralogist, 173, 1123-1133.

[30] Papike, J.J., Cameron, K.L. and Baldwin, K. (1974) Amphiboles and Pyroxenes: Characterization of Other than Quadrilateral Components and Estimates of Ferric Iron from Microprobe Data. Geological Society of America, Abstracts with Progrrams, 6, 1053-1054.

[31] Schweitzer, E.L., Papike, J.J. and Bence, A.E. (1979) Statistical Analysis of Clinopyroxenes from Deep-Sea Basalts. American Mineralogist, 64, 501-513.

[32] Stormer, J.C. (1983) The Effects of Recalculation on Estimates of Temperature and Oxygen Fugacity from Analyses of Multicomponent Iron-Titanium Oxides. American Mineralogist, 68, 586-594.

[33] Carmichael, L.S.E. (1967) The Iron-Titanium Oxides of Salic Volcanic Rocks and Their Associated Ferromagnesian Silicates. Contributions to Mineralogy and Petrology, 14, 36-64. https://doi.org/10.1007/BF00370985

[34] Buddington, A.F. and Lindsley, D.H. (1964) Iron-Titanium Oxide Minerals and Synthetic Equivalents. Journal of Petrology, 5, 310-357.

https://doi.org/10.1093/petrology/5.2.310

[35] Smith, J.V. and Brown, W.L. (1988) Feldspar Minerals. Second Revised and Extended Edition. Springer Verlag, Berlin.

[36] Frey, F.A., Green, D.H. and Roy, S.D. (1978) Integrated Models of Basalts Petrogenesis: A Study of Quartz Tholeiites to Olivine Melilites from South Eastern Australia Utilizing Geochemical and Experimental Petrological Data. Journal of Petrology, 19, 463-513. https://doi.org/10.1093/petrology/19.3.463

[37] Jung, S. and Masberg, P. (1998) Major and Trace Element Systematics and Isotope Geochemistry of Cenozoic Mafic Volcanic from the Vogelsberg (Central Germany): Constraints on the Origin of Continental Alkaline and Tholeiitic Basalts and Their Mantle Sources. Journal of Volcanology and Geothermal Research, 86, 151-177. https://doi.org/10.1016/S0377-0273(98)00087-0

[38] Maury, R.C. Brousse, R., Villemant, B., Joron, J.-L., Jaffrezic, H. and Treuil, M. (1980) Cristallisation fractionnée d'un magma basaltique alcalin: La série de la Chaîne des Puys (Massif central. France) I. Pétrologie. Bulletin de Minéralogie, 103, 250-266. https://doi.org/10.3406/bulmi.1980.7404

[39] Righter, K. and Rosas-Elguera, J. (2001) Alkaline Lavas in the Volcanic Front of the Western Mexican Volcanic Belt: Geology and Petrology of the Ayutla and Tapalpa Volcanic Fields. Journal of Petrology, 42, 2333-2361.

https://doi.org/10.1093/petrology/42.12.2333

[40] Jankovics Éva, M., Dobosi, G., Embey-Isztin, A., Kiss, B., Sági, T., Harangi, S. and Ntaflos, T. (2013) Origin and Ascent History of Unusually Crystal-Rich Alkaline Basaltic Magmas from the Western Pannonian Basin. Bulletin of Volcanology, 75, 2-23. https://doi.org/10.1007/s00445-013-0749-7

[41] Tracy, R.J. and Robinson, P. (1977) Zoned Titanian Augite in Alkali Olivine Basalt 
from Tahiti and the Nature of Titanium Substitutions in Augite. American Mineralogy, 62, 634-645.

[42] Wass, S.Y. (1979) Multiple Origins of Clinopyroxenes in Alkali Basaltic Rocks. Lithos, 12, 115-132. https://doi.org/10.1016/0024-4937(79)90043-4

[43] Dobosi, G., Schultz-Güttler, R., Kurat, G. and Kracher, A. (1991) Pyroxene Chemistry and Evolution of Alkali Basaltic Rocks from Burgenland and Styria, Austria. Mineralogy and Petrology, 43, 275-292. https://doi.org/10.1007/BF01164531

[44] Bryan, W.B., Finger, I. and Chayes, F. (1969) Estimation Proportions in Petrographic Mixing Equations by Least-Square Approximation. Science, 163, 926-927. https://doi.org/10.1126/science.163.3870.926

[45] Stormer, J.C. and Nicholls, J. (1978) XLFRAC: A Program for the Interactive Testing of Magmatic Differentiation Models. Computer and Geoscience, 4, 143-159. https://doi.org/10.1016/0098-3004(78)90083-3

[46] Morgan, D.J., Blake, S., Rogers, N.W., Devivo, B., Rolandi, G., MacDonald, R. and Hawkesworth, C.J. (2004) Time Scales of Crystal Residence and Magma Chamber Volume from Modelling of Diffusion Profiles in Phenocrysts: Vesuvius 1944. Earth and Planetary Sciences Letter, 222, 933-946.

https://doi.org/10.1016/j.epsl.2004.03.030

[47] Kahl, M., Chakraborty, S., Costa, F. and Pompilio, M. (2011) Dynamic Plumbing System beneath Volcanoes Revealed by Kinetic Modeling, and the Connection to Monitoring Data: An Example from Mt. Etna. Earth and Planetary Sciences Letter, 308, 11-22. https://doi.org/10.1016/j.epsl.2011.05.008

[48] Saunders, K.E., Blundy, J.D., Dohmen, R.G. and Cashman, K.V. (2012) Linking Petrology and Seismology at an Active Volcano. Science, 336, 1023-1027. https://doi.org/10.1126/science.1220066 